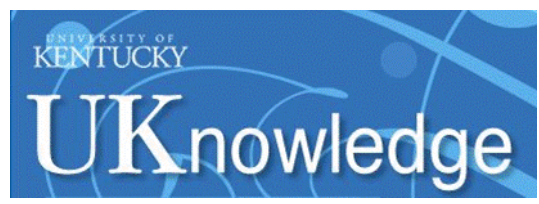

University of Kentucky

UKnowledge

9-24-2018

\title{
Continuum and Spectral Line Radiation from a Random Clumpy Medium
}

John E. Conway

Chalmers University of Technology, Sweden

Moshe Elitzur

University of Kentucky, moshe@uky.edu

Rodrigo Para

European Southern Observatoru, Chile

Follow this and additional works at: https://uknowledge.uky.edu/physastron_facpub

Part of the Astrophysics and Astronomy Commons

Right click to open a feedback form in a new tab to let us know how this document benefits you.

\section{Repository Citation}

Conway, John E.; Elitzur, Moshe; and Para, Rodrigo, "Continuum and Spectral Line Radiation from a Random Clumpy Medium" (2018). Physics and Astronomy Faculty Publications. 608.

https://uknowledge.uky.edu/physastron_facpub/608

This Article is brought to you for free and open access by the Physics and Astronomy at UKnowledge. It has been accepted for inclusion in Physics and Astronomy Faculty Publications by an authorized administrator of UKnowledge. For more information, please contact UKnowledge@lsv.uky.edu. 


\section{Continuum and Spectral Line Radiation from a Random Clumpy Medium}

Digital Object Identifier (DOI)

https://doi.org/10.3847/1538-4357/aadcf9

Notes/Citation Information

Published in The Astrophysical Journal, v. 865, no. 1, 70, p. 1-17.

(c) 2018. The American Astronomical Society. All rights reserved.

The copyright holder has granted the permission for posting the article here.

This article is available at UKnowledge: https://uknowledge.uky.edu/physastron_facpub/608 


\title{
Continuum and Spectral Line Radiation from a Random Clumpy Medium
}

\author{
John E. Conway ${ }^{1}$, Moshe Elitzur ${ }^{2,3}$ (10, and Rodrigo Parra ${ }^{4}$ \\ ${ }^{1}$ Department of Space, Earth and Environment, Chalmers University of Technology Onsala Space Observatory, SE-439 92 Onsala, Sweden \\ 2 Department of Physics and Astronomy, University of Kentucky, Lexington, KY 40506, USA \\ ${ }^{3}$ Astronomy Department, University of California, Berkeley, CA 94720, USA \\ ${ }^{4}$ European Southern Observatory, Chile \\ Received 2018 July 12; revised 2018 August 21; accepted 2018 August 22; published 2018 September 24
}

\begin{abstract}
We present a formalism for continuum and line emission from random clumpy media together with its application to problems of current interest, including $\mathrm{CO}$ spectral lines from ensembles of clouds and radio emission from $\mathrm{H}$ II regions, supernovae, and star-forming regions. For line emission, we find that the effects of clump opacity on observed line ratios can be indistinguishable from variations of intrinsic line strengths, adding to the difficulties in determining abundances from line observations. Our formalism is applicable to arbitrary distributions of cloud properties, provided the cloud volume filling factor is small; numerical simulations show it to hold up to filling factors of $\sim 10 \%$. We show that irrespective of the complexity of the cloud ensemble, the radiative effect of clumpiness can be parameterized at each frequency by a single multiplicative correction to the overall optical depth; this multiplier is derived from appropriate averaging over individual cloud properties. Our main finding is that cloud shapes have only a negligible effect on radiation propagation in clumpy media; the results of calculations employing point-like clouds are practically indistinguishable from those for finite-sized clouds with arbitrary geometrical shapes.
\end{abstract}

Key words: H II regions - ISM: abundances - line: formation - line: profiles - radiative transfer supernovae: general

\section{Introduction}

Radiation propagation in a nonuniform clumpy medium is a common problem in astrophysics. Example continuum applications include IR dust emission from circumnuclear tori in active galactic nuclei (AGNs; Nenkova et al. 2002, 2008), free-free absorption affecting supernova radio light curves and spectra (Weiler et al. 2004), radio-millimeter wave thermal emission from single massive stars (Ignace \& Churchwell 2004), and star formation-induced radio synchrotron emission accompanied by free-free absorption in galaxies (Lacki 2013). Spectral line applications include modeling the optical and UV spectra from AGN broad-line regions (Laor et al. 2006) and interstellar atomic and molecular lines (Martin et al. 1984; Wall 2006, 2007). A common approach to the analysis of emission from clumpy media, pioneered by Martin et al. (1984), is to assume some geometry for the individual clouds and proceed by averaging over properties along the line of sight (LOS). Some general scaling relations emerged in these works, but they remained unexplained. In particular, Ignace \& Churchwell (2004) noted that, in their modeling based on spherical clouds, only the distribution in individual cloud optical depths could affect the spectral shape-the cloud radii were irrelevant.

In an entirely different approach, Natta \& Panagia (1984) modeled clumpy media absorption with point-like identical structureless absorbers characterized by a single property, an optical depth, and no other parameters. Noting that random placement yields a Poisson distribution for the number of absorbers along the LOS, Natta \& Panagia derived the effective optical depth of the medium from the mean number of absorbers along the LOS and their common optical depth. Nenkova et al. $(2002,2008)$ extended this formalism to the expected emission from a population of such clouds and placed the Natta \& Panagia point-like absorbers concept on a more solid footing by showing that the ratio of cloud size to the mean free path between clouds is equal to $\phi$, the cloud volume filling factor. Therefore, when $\phi \ll 1$, each cloud appears as a point from its nearest neighbor; thus, its geometry can be ignored. Still, the usage of a single optical depth per absorber remained problematic. For example, in the case of a sphere with optical depth $\tau$ along the diameter, the actual optical depth along an LOS can vary from $\tau$ for an LOS through the center to zero for a grazing LOS. Here we address this issue, bringing the Natta \& Panagia formalism to completion.

Starting in Section 2, we generalize both clumpy absorption and emission to an arbitrarily complex mixture of clump properties, including variations of these properties along the LOS. The only restrictions are that the medium is random (i.e., cloud positions are uncorrelated) and the propagating radiation does not affect the cloud absorption and emission properties. We investigate via Monte Carlo simulations the range of volume filling factors over which our formalism applies and show that significant departures occur only at relatively large filling factors $(\phi \gtrsim 0.1$; Section 2.1), introduce the concept of a clumping factor that modulates the effective opacity of a clumpy medium and depends on the average properties of its clumps (Section 2.2), and extend the clump formalism to include spectral line absorption (Section 2.3) and emission (Section 2.4). Section 3 explores the effects of cloud shapes and shows that they have no significant impact on radiation propagation in a clumpy medium. In Section 4, we apply our formalism to a couple of current problems involving clumpy emission and absorption by continuum and spectral lines; Appendix C provides some additional examples. Section 5 contains a summary and discussion.

\section{Radiation Transfer in Clumpy Media}

Consider a region where matter is concentrated within clouds that occupy a fraction $\phi$ of the overall volume. The medium 
will be regarded as clumpy whenever the filling factor obeys $\phi \ll 1$. This condition is mathematically equivalent to the requirement that the size of individual clouds is much smaller than the mean free path between them (Nenkova et al. 2008). In that case, each cloud can be considered a "mega-particle"-a point characterized by its radiative properties but whose shape and size are irrelevant. With this assumption, and taking all clouds to be identical, Natta \& Panagia (1984) derived the effective optical depth at frequency $\nu$ along any LOS through a clumpy medium in terms of the individual cloud optical depth, $\tau_{\nu}$, and the mean number of clouds along the LOS, $\mathcal{N}$ (see Appendix A.1).

In Appendix A.2, we generalize the Natta \& Panagia result (Equation (22)) to an arbitrary mixture of cloud types and show that radiation propagating through a clumpy medium will have a mean transmission factor ${ }^{5}$ of $\exp \left(-\tau_{\mathrm{E} \nu}\right)$, where

$$
\tau_{\mathrm{E}_{\nu}}=\mathcal{N}\left(1-\left\langle e^{-\tau_{\nu}}\right\rangle\right)
$$

is the effective optical depth as a function of frequency. In this expression, $\left\langle e^{-\tau_{\nu}}\right\rangle$ is the mean transmission factor for radiation passing through a single cloud, where the averaging is done over all cloud types. This result reverts to the Nattta \& Panagia expression when all clouds are identical but otherwise generalizes it in two fundamental aspects. (1) Even for pointlike, structureless clouds characterized only by optical depth, $\tau_{\nu}$ can still vary from cloud to cloud; Equation (1) shows that such variations can be handled by simply averaging the transmission factor $e^{-\tau_{\nu}}$ over all clouds. (2) Actual clouds cannot really be characterized by the single parameter $\tau_{\nu}$. Because real clouds have geometrical shapes and sizes, the optical depth varies with the impact parameter of the LOS relative to the center of a spherical cloud, the orientation of a filamentary cloud, etc. (see Appendix B). Geometrical shape is an additional cloud property, easily incorporated by adding independent variables to describe the cloud distribution. Since the average in Equation (1) can be made over any number of parameters that describe the cloud population, when cloud placement is random, one can first average over all shape-related parameters for each cloud type before averaging over the range of cloud types in the population (for example, with each cloud type perhaps having a different mean or peak opacity).

The above analysis for absorption is easily generalized to that of emission from a clumpy medium. Nenkova et al. (2002, 2008) derived the expression for a single type of cloud, and Appendix A.3 generalizes their analysis to the case of a cloud distribution. The emerging mean emission along an LOS through a clumpy medium is

$$
I_{\mathrm{C} \nu}=\int e^{-\tau_{E \nu}(s)}\left\langle S_{\mathrm{C} \nu}(s)\right\rangle N(s) d s .
$$

In this expression, $S_{\mathrm{C} \nu}(s)$ is the source function for single-cloud emission at position $s$ along the LOS and $\left\langle S_{\mathrm{C} \nu}(s)\right\rangle$ is its average over all cloud types at $s$ (see Equation (27)), $N(s)$ is the mean number of clouds per unit length at that point (see Appendix A.1), and $\tau_{\mathrm{E}_{\nu}}(s)$ is the effective optical depth, defined as in Equation (1), from $s$ to the edge of the source.

\footnotetext{
5 When averaging over a telescope beam containing many independent LOSs sharing the same cloud properties, the measured value of the transmission factor is expected to lie close to this quantity, which is formally the statistically average transmission factor for each LOS.
}

\subsection{Effects of Volume Filling Factor}

The only properties of the cloud distribution that enter into Equations (1) and (2) are the mean number of clouds per LOS, $\mathcal{N}$, and the number of clouds per unit length, $N(s)$. There is no dependence on the cloud volume filling factor $\phi$; by assuming $\phi \ll 1$, we ended up with results that are entirely independent of the volume filling factor. The reason is simple: a complete formalism, in which one would not invoke the assumption $\phi \ll 1$ from the start, would lead to a series expansion in powers of $\phi$. The expressions derived above are simply the zeroth-order terms in that expansion; i.e., they are the $\phi \rightarrow 0$ limit of a more complete formalism (see also Nenkova et al. 2008).

Assuming $\phi \ll 1$ at the outset precludes us from studying the effects of finite volume filling factors and the limitations of our formalism. To try to quantify the finite- $\phi$ corrections, we have carried out numerical Monte Carlo simulations for the simple case of identical clouds to determine the critical volume filling factor at which significant deviations from our theory occur. Our simulations further assume clouds that are spherical and uniform and hence fully characterized by a single number, $\tau_{1}$, the optical depth across their diameters. ${ }^{6}$ We investigated several values of $\tau_{1}$ and, for each one, varied the mean number of clouds per LOS $\mathcal{N}$ and volume filling factor $\phi$ by filling a cube with side $2 L$ with $N_{\text {tot }}$ spheres of radius $R$ such that

$$
\frac{R}{L}=\frac{3}{4} \frac{\phi}{\mathcal{N}}, \quad N_{\text {tot }}=\frac{8}{\pi}\left(\frac{L}{R}\right)^{2} \mathcal{N} \text {. }
$$

We carried out a large number of Monte Carlo simulations, varying $R / L$ and $N_{\text {tot }}$ (i.e., $\mathcal{N}$ and $\phi$ ) and averaging the results. The simulation results were then compared with the $\phi \rightarrow 0$ theory. Figure 1 presents the comparison, showing that Equation (1) adequately describes volume filling factors as large as $10 \%$, for which the mean transmission factors $\exp \left(-\tau_{\mathrm{E}}\right)$ obtained from that expression (see Equation (35b), Appendix B) and the Monte Carlo simulations agree to within $15 \%$ at all optical depths. For the particular case of optically thin clouds, illustrated by the $\tau_{1}=0.1$ case shown in the left panel, the Monte Carlo simulations produce close agreement with theory at all the tested volume filling factors. This is as expected because, as shown below (Section 2.2), clumping is irrelevant when individual clouds are optically thin. In this pseudo-continuous-medium case, the effective absorption depends only on $\mathcal{N} \tau_{1}$, the overall mean optical depth along the LOS. The clumpy nature of the medium becomes important only when individual clouds are optically thick, as seen from the figure's two other panels. In this case, deviations from the analytic results occur at high filling factors, where Equation (1) overpredicts the fraction of transmitted radiation (i.e., underpredicts the effective opacity). The reason is that large volume filling factors involve large cloud sizes, and keeping the clouds from interpenetrating each other produces significant deviations from Poisson statistics, leading to narrower probability distributions for the numbers of clouds per LOS. The deviations from the analytic $\phi \rightarrow 0$ limit are noticeable in the middle panel, which shows results for $\tau_{1}=1$,

\footnotetext{
6 Since we consider only absorption at a single frequency, here and in what follows, we drop the subscript $\nu$.
} 


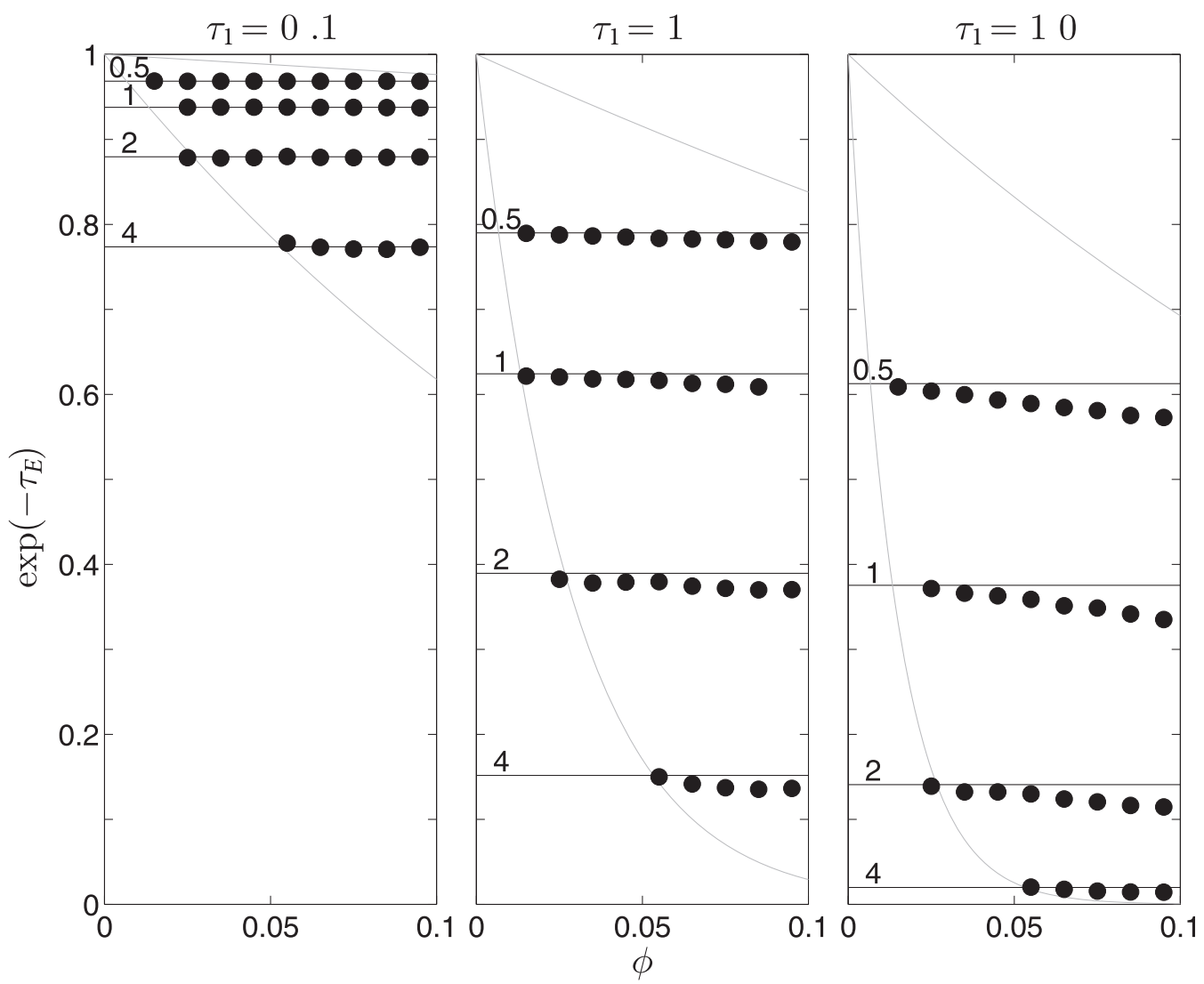

Figure 1. Results of Monte Carlo simulations to investigate departures at large volume filling factors $(\phi)$ from the predictions of the $\phi \rightarrow 0$ analytic clump formalism. Each panel shows the expectation value of the attenuation of background radiation, $\exp \left(-\tau_{\mathrm{E}}\right)$, by a volume containing randomly placed identical spherical absorbing clouds. The three panels show results for clouds with different optical depth $\tau_{1}$ across the diameter, as marked. The right panel $\tau_{1}=10$ simulations are representative of all $\tau_{1} \geqslant 10$. In each panel, horizontal lines show the $\phi \rightarrow 0$ analytic predictions from Equation (1) for transmission factors for differing mean numbers of clouds per LOS, as marked $(\mathcal{N}=0.5,1,2$, and 4). Filled circles show the results of numerical Monte Carlo simulations for the transmission factor as a function of $\phi$ for each $\mathcal{N}$. The statistical error bars on these simulations are comparable to the size of the circles. Gray curved lines trace the boundaries of the simulations; the left one is the locus of cloud radius $1 \%$ the side length of the control volume, and the right one is $20 \%$ (cf. Equation (3)). Significant departures from the $\phi=0$ analytic expression are seen only for optically thick clouds and never exceed $\sim 15 \%$ as long as $\phi \leqslant 0.1$.

and are even more pronounced in the right panel, where $\tau_{1}=10$. Still, these deviations never exceed $15 \%$ in any of the simulated cases. Further increasing the single-cloud optical depth has no effect on the outcome because clouds with $\tau_{1}=10$ are already completely opaque; hence, the deviations seen in the figure's right panel apply to all $\tau_{1} \geqslant 10$.

These results show that Equation (1) is an excellent approximation for practical applications. In all cases that we have simulated, we find that, up to $\phi=0.1$, this analytic expression correctly predicts the absorption by the cloud ensemble to better that $15 \%$. We expect that the critical volume filling factor found in these simulations will also apply to more complex cases involving a mixture of cloud types. Such mixtures will include values of $\tau_{1}$ that are both larger and smaller than 10. As noted above, clouds with $\tau_{1}>10$ will induce the same deviations from the $\phi=0$ limit as the purely $\tau_{1}=10$ case. And since such deviations are even smaller for the mixture clouds that have $\tau_{1}<10$, the model with $\tau_{1}=10$ for all clouds provides the maximal deviations from the analytic result in Equation (1).

\subsection{The Clumping Correction Factor}

Equation (1) gives the value of the effective optical depth of a clumpy medium $\tau_{\mathrm{E} \nu}$, such that $\exp \left(-\tau_{\mathrm{E} \nu}\right)$ is the expectation or mean value of the transmission of background radiation. In contrast, the mean of the total optical depth through the source is

$$
\tau_{\mathrm{T}_{\nu}}=\mathcal{N}\left\langle\tau_{\nu}\right\rangle .
$$

This quantity would be the overall optical depth if the clouds were dispersed into a smooth medium maintaining the same total gas column density along the LOS. Therefore, the ratio

$$
K_{\nu} \equiv \frac{\tau_{\mathrm{E}_{\nu}}}{\tau_{\mathrm{T}_{\nu}}}
$$

measures the factor by which the clumpy medium effective opacity is reduced compared to its total opacity due to the effects of clumping. From Equations (1) and (4),

$$
K_{\nu}=\frac{1-\left\langle e^{-\tau_{\nu}}\right\rangle}{\left\langle\tau_{\nu}\right\rangle} .
$$

The functional form of this clumping correction factor resembles the escape probability familiar from line transfer calculations. When $\tau_{\nu} \ll 1$ for every cloud, i.e., all clouds are optically thin, $K_{\nu}=1$ : the effective optical depth equals the total optical depth at the given frequency, and clumping is irrelevant. Note that the only requirement for this condition to be met is that individual clouds be optically thin; the total optical depth, $\mathcal{N}\left\langle\tau_{\nu}\right\rangle$, can still be large. If we keep $\tau_{\mathrm{T}_{\nu}}$ fixed and 
increase the number of clouds $\mathcal{N}$ along the path, individual clouds will decrease in opacity until they become optically thin when $\mathcal{N}>\tau_{\mathrm{T}_{\nu}}$, yielding $K_{\nu} \simeq 1$; that is, for fixed $\tau_{\mathrm{T}_{\nu}}$, the absorption properties approach the smooth-density limit at large (generally frequency-dependent) $\mathcal{N}$ even if the cloud volume filling factor remains small. On the other hand, when $\tau_{\nu} \gg 1$ for every cloud, $K_{\nu}=1 /\left\langle\tau_{\nu}\right\rangle$, and the effective opacity is less than that for a smooth medium of the same mean column density. In this case, $\tau_{\mathrm{E}_{\nu}}=\mathcal{N}$, meaning that although individual clouds are optically thick, a photon still escapes when it avoids all clouds along the LOS, an event whose probability according to Poisson statistics is $e^{-\mathcal{N}}$.

Since $K_{\nu}$ is always $\leqslant 1$, clumping can only decrease the effective opacity, reflecting the possibility of photon escape even when all clouds are optically thick. Significantly, $K_{\nu}$ is determined only by the mean single-cloud optical depth, independent of the number of clouds. Since the effective opacity obeys $\tau_{\mathrm{E}_{\nu}}=K_{\nu} \tau_{\mathrm{T}_{\nu}}$, we can use $\tau_{\mathrm{T}_{\nu}}$ and $K_{\nu}$ to parameterize a region's absorption properties instead of $\tau_{\nu}$ and $\mathcal{N}$. Sources with the same $\tau_{\mathrm{T}_{\nu}}$ and $K_{\nu}$ will have identical absorption properties even if their clouds have very different shapes and distributions in properties.

\subsection{Line Absorption by Clouds in Motion}

The formalism developed above is applicable to the transfer of radiation at frequency $\nu$ no matter how the optical depth $\tau_{\nu}$ varies with frequency. Bulk cloud motions introduce Doppler shifts that couple velocity and frequency distributions. This coupling can be ignored in the case of continuum radiation, where the variation of $\tau_{\nu}$ is negligible for any of the Doppler shifts. Lines are at the opposite limit-a spectral line has $\tau_{\nu}=\tau_{0} \Phi\left(\nu-\nu_{0}\right)$, where $\tau_{0}$ is the optical depth at the linecenter frequency $\nu_{0}$ and $\Phi$ is sharply peaked about that frequency. We now show that thanks to its narrow spectral range, the whole line can be treated as a single entity rather than frequency by frequency.

Replacing the frequency shift from line center by the equivalent Doppler velocity $v$, the line profile is $\Phi(v)$, with normalization chosen such that $\Phi(0)=1$. The cloud velocity width can then be defined as $\Delta v=\int \Phi(v) d v$, a quantity that characterizes the range of internal LOS motions within the cloud. ${ }^{7}$ Note that the ensemble of clouds encountered along an LOS can have a range of different velocity widths $\Delta v$ or linecenter opacities $\tau_{0} .{ }^{8}$ Invariably, the observed ensemble also contains clouds with a range of different bulk Doppler velocities, $N(u)$ being the number of clouds encountered on average along the radiation path per unit LOS velocity at bulk Doppler velocity $u$ (see Appendix A.1). Bulk cloud motions strongly affect the line propagation when their range is much larger than $\Delta v$. Appendix A.4 shows that the effective optical depth is given by the convolution equation

$$
\tau_{\mathrm{E}}(v)=\int N(u)\left(1-\left\langle e^{-\tau(u-v)}\right\rangle\right) d u,
$$

where the angle brackets denote averages over all cloud properties other than their LOS velocity. Since the optical

\footnotetext{
7 A Gaussian Doppler profile $\Phi(v)=\exp \left(-v^{2} / \sigma^{2}\right)$ gives $\Delta v=\sqrt{\pi} \sigma$.

8 Line-center opacities $\tau_{0}$ can vary between different kinds of clouds within the ensemble. Even for identical clouds, they can still vary due to encountering a cloud with a different LOS impact parameter or orientation.
}

depth across a spectral line profile vanishes rapidly when $|u-v|>\Delta v$, the contributions to the convolution integral are confined mostly to a narrow interval with width $\sim \Delta v$ around the LOS velocity $u=v$. When $N(u)$ varies only on scales much larger than $\Delta v$, it can be taken as constant across the line profile. Then, to a good degree of approximation, the effective optical depth at $v$, the line-center Doppler shift from rest frequency, is

$$
\begin{aligned}
\tau_{\mathrm{E}}(v) & =N(v)\langle W\rangle(v), \\
\text { where }\langle W\rangle(v) & =\int\left(1-\left\langle e^{-\tau(u-v)}\right\rangle\right) d u
\end{aligned}
$$

is the velocity equivalent width of the mean normalized emission profile of single clouds averaged over all cloud types at spectral velocity $v$. In this general case, a $v$-dependence of $\langle W\rangle$ may occur when typical cloud peaks $\tau_{0}$ and/or $\Delta v$ vary with the cloud bulk velocity; for consistency of the derivation, such variations must occur over velocity scales much larger than $\Delta v$. When the cloud line shapes and opacities do not depend on the cloud velocities, the $v$-dependence can be dropped, and we can consider a single value of the equivalent width $\langle W\rangle$.

Following the definition given in Section 2.2, the total optical depth for spectral line emission at velocity $v$ is, on average, $\int N(u)\langle\tau(u-v)\rangle d u \approx N(v) \int\langle\tau(u-v\rangle d u$. From our definition of $\Delta v, \int\langle\tau(u-v)\rangle d u=\left\langle\tau_{0} \Delta v\right\rangle(v)$, where the averaging is made over all properties of the ensemble clouds other than bulk velocity. Therefore,

$$
\tau_{\mathrm{T}}(v)=N(v)\left\langle\tau_{0} \Delta v\right\rangle(v) .
$$

This would be the total optical depth if the absorbing particles in all clouds along a given LOS were dispersed into a spatially smooth distribution. The correction factor converting total line opacity into effective clumpy line opacity is thus

$$
K_{\text {line }}(v) \equiv \frac{\tau_{\mathrm{E}}(v)}{\tau_{\mathrm{T}}(v)}=\frac{\langle W\rangle(v)}{\left\langle\tau_{0} \Delta v\right\rangle(v)},
$$

the line equivalent of the single-frequency clumping factor defined by Equation (5). Line emission behaves the same as continuum: the effect of an arbitrarily complex clumpy distribution again is reduced to a multiplicative factor determined from the properties of individual clouds and independent of their number. At every spectral velocity $v$, the entire line is described by the single clumping correction factor $K_{\text {line }}(v)$. When cloud properties are independent of bulk velocity, a single multiplicative factor links effective and total opacities at all line velocities.

When every cloud is optically thin at line center, $\langle W\rangle(v) \approx \int\langle\tau(u-v)\rangle d u=\left\langle\tau_{0} \Delta v\right\rangle(v) \quad$ Equation (8)) so that $K_{\text {line }}=1$. When $\tau_{0}>1$ for every cloud, the velocity integration that determines $\langle W\rangle$ can be approximated as effectively truncated at $u=\delta v_{\tau_{0}}$, the velocity that separates the optically thick core from the optically thin line wings. For the Doppler profile $\delta v_{\tau_{0}} \propto \Delta v \sqrt{\ln \tau_{0}}$, yielding the large- $\left\langle\tau_{0}\right\rangle$ behavior $K_{\text {line }} \sim \sqrt{\ln \left\langle\tau_{0}\right\rangle} /\left\langle\tau_{0}\right\rangle$. In contrast, single-frequency clumping produces $K_{\nu} \propto 1 /\left\langle\tau_{\nu}\right\rangle$ at large $\left\langle\tau_{\nu}\right\rangle$ (see Section 2.2), a steeper decline than that of $K_{\text {line }}$ (see also Figure 2(b) below). The reason for the flatter asymptotic behavior of $K_{\text {line }}$ is that 

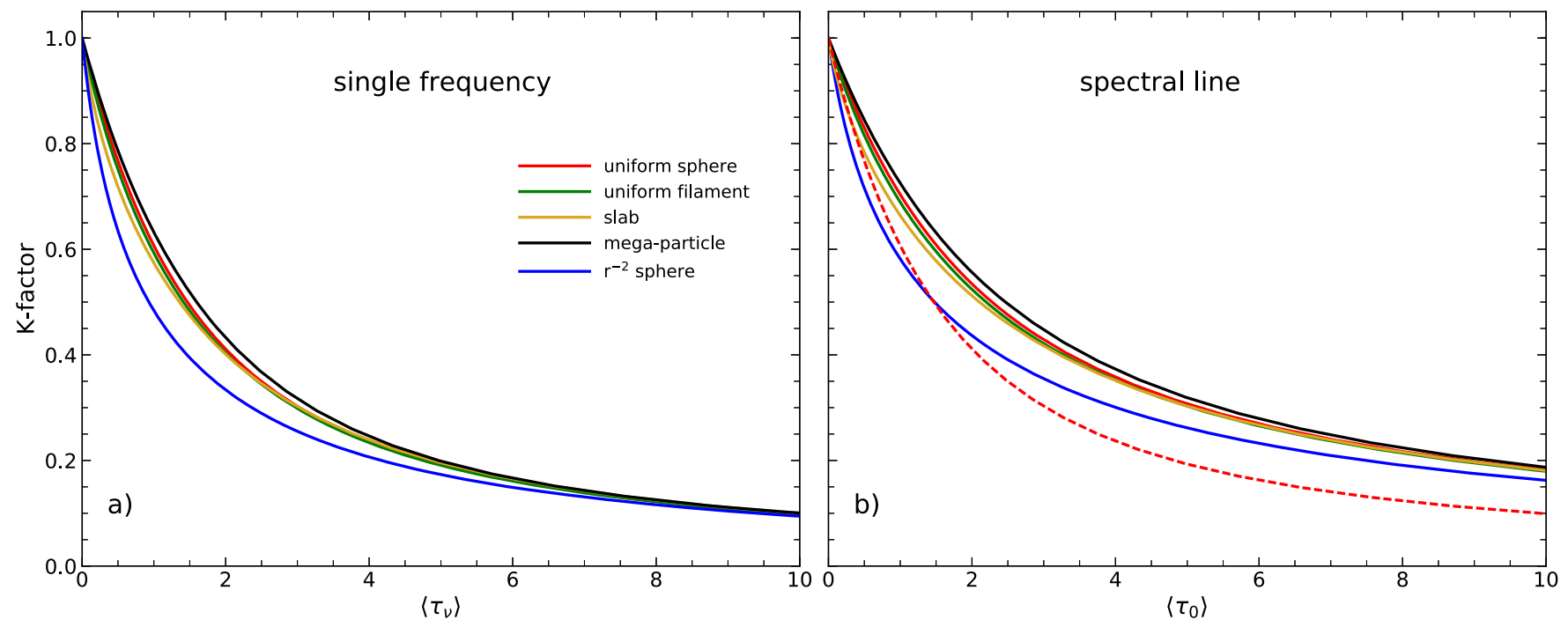

Figure 2. Effect of cloud geometrical shape. The clumping correction $K$-factor as a function of the cloud mean optical depth (a) at a single frequency (Equation (6); relevant to the continuum case) and (b) for a spectral line (Equation (10)), where $\left\langle\tau_{0}\right\rangle$ is the average optical depth at line center. Different cloud geometries are plotted with different colors, as labeled. A "mega-particle" is a structureless absorber whose only property is optical depth $\tau_{\nu}$; the $r^{-2}$ sphere is a spherical cloud with absorption coefficient $\propto r^{-2}$ (see text for details). For comparison, the dashed line in the right panel reproduces the uniform-sphere plot from the left panel. Note the relatively weak dependence of $K$ on geometry, especially for the slab and the uniform sphere and filament.

clumpiness has only a negligible effect in the line wings, which are optically thin even when the cloud is optically thick at the line center. Thus, the overall clumping correction effect is less significant for the line than for the transfer of continuum radiation at the line-center frequency through a clumpy medium with the same total optical depth.

\subsection{Spectral Line Emission}

The emission from a population of line-emitting clouds can be calculated following the steps outlined for the continuum case in Section 2. The details are given in Appendix A.5, where it is shown that Equation (2) is applicable as is, in unmodified form, to the case of line intensity emerging from a clumpy medium. This relation is the equivalent of the standard formal solution of the radiative transfer equation for clumpy media, with the appropriate attenuation term for continuum and line radiation. The only slight complication for the lines is the two-step averaging in Equation (32), which involves also averaging over the spectral shape of single-cloud emission (Equation (30)) to get the local average cloud source function. Equation (2) holds at every frequency/velocity for line radiation whenever the ensemble averages for the cloud emission and absorption properties are independent of each other, i.e., when the optical depths of individual clouds are unaffected by the propagating line radiation. When this condition is violated, the intensity calculation requires an iterative procedure based on the expressions given here. Details of an iteration scheme analogous to standard $\Lambda$ iterations are described in Nenkova et al. 2008 (see Section 3.2 of that paper).

When we express intensity in brightness temperature units and use the common approximation of a constant line excitation temperature $T_{\mathrm{x}}$ inside each cloud, the brightness temperature $T_{\mathrm{b}}$ of the emergent radiation assumes the simple form of Equation (34). When the cloud velocity distribution is much broader than the velocity width of internal cloud motions, this becomes

$$
\begin{aligned}
T_{\mathrm{b}}(v) & =\left\langle T_{x}\right\rangle[1-\exp (-\langle W\rangle N(v))] \\
& =\left\langle T_{x}\right\rangle\left[1-\exp \left(-K_{\text {line }} \tau_{\mathrm{T}}(v)\right)\right],
\end{aligned}
$$

given in terms of either the mean cloud equivalent width $\langle W\rangle$ and the number of clouds per unit velocity $N(v)$ or the clumping correction factor $K_{\text {line }}$ and the total optical depth versus velocity $\tau_{\mathrm{T}}(v)$.

\section{Effects of Cloud Geometry}

Among the many variables affecting the radiative properties of a clumpy medium is the geometrical shape of the clouds. Our formalism shows that the shape affects radiation attenuation only through the clumping factor $K$, enabling us to study the impact of geometry. To separate the effect of shape from all other properties, we consider an ensemble of identical clouds. When the clouds are additionally taken to be uniform, the required averages involve only variation of length along the LOS through the cloud and thus depend on geometry alone and nothing else. Since a sphere is the ultimate isotropic shape, while an elongated filament and a semiinfinite slab are at the other extreme end of anisotropy, these geometries can be expected to bracket the full range of $K$-factor variation that cloud shapes can induce. Note, in particular, that in studies of AGNs, the slab geometry is invoked in all calculations of broad-line emission (see, e.g., Laor et al. 2006) and in some models of torus IR emission (Nenkova et al. 2008).

The averages involve integrations over the cloud projected area for a sphere, orientation for a slab, and both of them in the case of a filament; Appendix B gives the details. The left panel of Figure 2 shows the variation of $K_{\nu}$ with $\left\langle\tau_{\nu}\right\rangle$, the singlefrequency cloud mean optical depth relevant for continuum emission, for slabs (orange) and uniform spheres (red) and filaments (green). The three are hardly distinguishable from each other. This weak dependence on cloud geometrical shape is easy to understand: as noted above (Section 2.2), irrespective of geometry, $K$ is $\sim 1$ at small optical depths and $\sim 1 /\langle\tau\rangle$ at 
large ones. The geometrical shape affects only the transition between these two regimes, where its impact is constrained by the limits that must be joined on either end; Appendix B provides some further insight into this behavior.

The similarity of the results for slabs, spheres, and filaments suggests that all uniform clouds, whatever their geometry, have roughly the same $K$-factor when taken as a function of $\langle\tau\rangle$. To further test this possibility, we considered the extreme limit of "mega-particles," structureless clouds whose only property is optical depth $\tau_{\nu}$; then $\left\langle\tau_{\nu}\right\rangle=\tau_{\nu}$ and $K_{\nu}=\left(1-e^{-\tau_{\nu}}\right) / \tau_{\nu}$. Shown in black in Figure 2, the $K$-factor for a mega-particle closely resembles that for a sphere; the largest difference between the two is less than $5 \%$ (at $\langle\tau\rangle=2.2$ ).

These results show that the cloud shape plays only a minor role for uniform clouds. Internal structure within clouds adds another degree of freedom. To gauge its potential impact, the blue curve in Figure 2 shows $K_{\nu}$ for spherical clouds with an absorption coefficient proportional to $r^{-2}$ in the radius range $r_{\min } \leqslant r \leqslant 10 r_{\min }$. While this curve is significantly outside the narrow range covered by uniform clouds, the largest difference between the $r^{-2}$ sphere and a uniform one is still no more than $\sim 20 \%$ (at $\langle\tau\rangle=1.1$ ).

The right panel of Figure 2 shows the spectral line clumping factor $K_{\text {line }}$ (Equation (10)). For comparison, the singlefrequency $K_{\nu}$ for a uniform sphere is reproduced with a dashed line. At the same $\langle\tau\rangle, K_{\text {line }}$ is significantly larger than $K_{\nu}$. The reason is that, whatever the optical depth at line center, the wings of a spectral line always become optically thin at some point, and the effect of clumping disappears there. In addition, $K_{\text {line }}$ exhibits a flatter decline than $K_{\nu}$ at high optical depths-as expected, given that the large- $\langle\tau\rangle$ asymptotic behavior of $K_{\text {line }}$ is $\sqrt{\ln \left\langle\tau_{0}\right\rangle} /\left\langle\tau_{0}\right\rangle$, while that of $K_{\nu}$ is $1 /\left\langle\tau_{\nu}\right\rangle$ (see Section 2.3). Apart from these differences, the overall behavior of $K_{\text {line }}$ closely resembles that of $K_{\nu}$, similarly displaying only a weak dependence on geometry. Cloud shape is of secondary importance to radiation propagation in clumpy media.

\section{Example Applications}

In this section, we illustrate our general formalism for clumpy media by applying it to two concrete cases involving continuum and line emission. Some additional examples are provided in Appendix C.

\subsection{Radio Continuum Emission from Clumpy Ultracompact H II Regions}

Radio free-free emission from ultracompact $\mathrm{H}$ II regions (UCHIIs) often has a power-law spectrum, $I_{\nu} \propto \nu^{\alpha}$, with spectral index $\alpha \sim 1$ over a wide range of frequencies (Ignace $\&$ Churchwell 2004, hereafter IC04). Such spectra are too flat to be explained by optically thick free-free emission $(\alpha=2)$, too steep to be optically thin $(\alpha=-0.1)$, and extend over too wide a frequency range to be the transition between optically thin and thick emission from a single gas phase. Although smooth ionized outflow models can explain the observed spectra, IC04 argued that the wind properties become physically implausible for $\alpha \geqslant 1$. Therefore, IC04 instead modeled such radio-millimeter spectra by a clumpy medium in which the clumps had a wide distribution of emission measures (EMs). Specifically, they investigated the emission from an assembly of uniform spherical clouds. The free-free optical depth across the diameter of each cloud is

$$
\tau_{\nu}=\tau_{0}\left(\frac{\nu_{0}}{\nu}\right)^{2.1}
$$

where $\tau_{0}$ is the optical depth at some fiducial frequency $\nu_{0}$. The cloud number distribution as a function of $\tau_{0}$ was taken as a truncated power law between two limits:

$$
N\left(\tau_{0}\right) \propto \tau_{0}^{-\gamma}, \quad \tau_{0, \min } \leqslant \tau_{0} \leqslant \tau_{0, \text { max }} .
$$

As expected, IC04 found that the cloud ensemble spectrum had $\alpha=2$ at very low frequencies, where all clouds were optically thick, while at very high frequencies, where all clouds were optically thin, the spectral index was $\alpha=-0.1$. In between, over a broad region of frequencies in which some clouds were optically thick and some thin, there was an almost constant intermediate spectral index, $\alpha \approx 1$. Ignace \& Churchwell (2004) found that they could fit the spectra observed in UCHII regions assuming $\gamma \approx 1.5$ and a large ratio $\tau_{0, \max } / \tau_{0, \min }>100$. However, their computed spectra assumed that the overall mean number of clouds per LOS obeyed $\mathcal{N} \ll 1$; that is, they did not treat the effects of one cloud shadowing another.

While IC04 argued that cloud shadowing was of minor importance for the one source they modeled in detail (W49N $\mathrm{B} 2$, where $\mathcal{N} \approx 0.15$ ), it is clearly of interest to generalize their results to all values of $\mathcal{N}$. This can be readily done using the formalism developed here. The general expression for continuum emission from a clumpy medium is given by Equation (2). When both the mean cloud emissivity $\left\langle S_{\mathrm{C} \nu}\right\rangle$ and transmission factor $\left\langle e^{-\tau_{\nu}}\right\rangle$ are independent of position along the LOS, it is convenient to introduce the variable $t=\mathcal{N}(s) / \mathcal{N}$, the fraction of the total number of clouds along the LOS to position $s$ in the source (see Appendix A.1). Then $N(s) d s=\mathcal{N} d t$ and the $t$ integration is immediate, yielding

$$
I_{\mathrm{C} \nu}=\left\langle S_{\mathrm{C} \nu}\right\rangle \frac{1-e^{-\tau_{\mathrm{E} \nu}}}{1-\left\langle e^{-\tau_{\nu}}\right\rangle},
$$

where $\tau_{\mathrm{E}_{\nu}}$ is given by Equation (1). With this result, we can calculate the emerging spectrum from an ensemble of uniform spherical clouds similar to that investigated by IC04, but now removing the limitation $\mathcal{N}<1$.

We assume that each cloud emissivity is given by the Planck function $B_{\nu}(T)$, where $T$ is the cloud temperature, and then the cloud contribution to the specific intensity of the ensemble is $B_{\nu}(T)\left(1-e^{-\tau_{\nu}}\right)$, where $\tau_{\nu}$ is the cloud optical depth along the impact parameter corresponding to a particular LOS. To form $\left\langle S_{\mathrm{C} \nu}\right\rangle$, we must average over the cloud ensemble, i.e., cloud opacities and temperatures, as well as LOS impact parameter. Assuming that $T$ and $\tau_{0}$ are uncorrelated and utilizing the Rayleigh-Jeans limit for $B_{\nu}$ in the radio regime yields $\left\langle S_{\mathrm{C} \nu}\right\rangle=\left[2 k\langle T\rangle \nu^{2} / c^{2}\right]\left(1-\left\langle e^{-\tau_{\nu}}\right\rangle\right)$. In this expression, the average for the self-absorption factor is taken over both the cloud LOS impact parameter and the $\tau_{0}$ distribution. Substituting in Equation (14), we get

$$
I_{\mathrm{C} \nu}=\frac{2 k\langle T\rangle \nu^{2}}{c^{2}}\left(1-e^{-\tau_{\mathrm{E} \nu}}\right) .
$$

This is the same expression as for emission from a single cloud, except that the cloud temperature is replaced by the ensemble average $\langle T\rangle$ and its optical depth $\tau_{\nu}$ is replaced by $\tau_{\mathrm{E}_{\nu}}$, the effective optical depth of the cloud distribution at each 


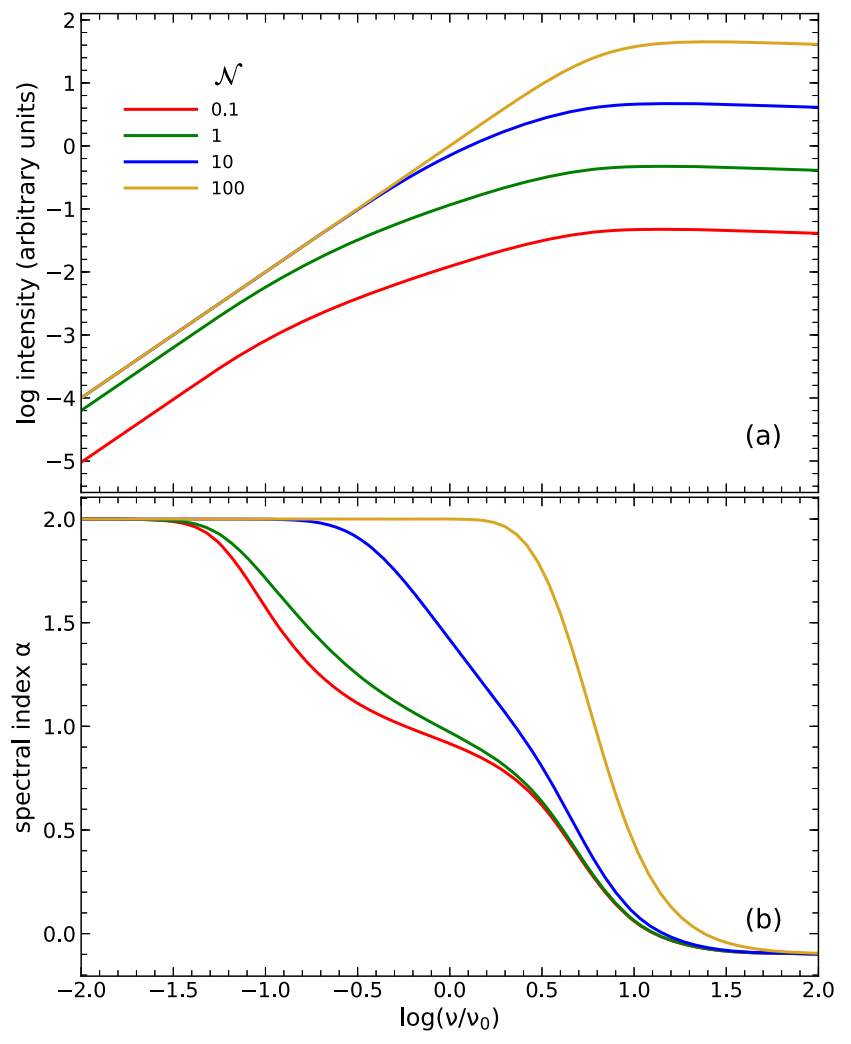

Figure 3. Free-free emission from a cloud ensemble with a wide range of optical depths in a truncated power-law distribution (Equation (13)). (a) Emerging spectra for various values of $\mathcal{N}$, the mean number of clouds along the LOS, as marked, and (b) the corresponding spectral index $\alpha=d \ln I_{\nu} / d \ln \nu$. The resulting spectral indices span the range between the optically thick $(\alpha=2)$ and thin $(\alpha=-0.1)$ limits for single-cloud emission. The $\mathcal{N}=0.1$ case (red curves), which is representative of all $\mathcal{N} \ll 1$, shows a significant frequency range over which $\alpha$ is close to unity, but this range narrows as $\mathcal{N}$ increases.

frequency. This latter quantity, in turn, is given by Equation (1), which can be written $\tau_{\mathrm{E}_{\nu}}=\mathcal{N} f(\nu)$, where $f(\nu) \equiv 1-\left\langle e^{-\tau_{\nu}}\right\rangle$ is the average absorption fraction of radiation at frequency $\nu$ encountering a cloud in the distribution; this quantity is determined purely by the mean properties of individual clouds and is independent of the number of clouds.

Using Equation (15), we can estimate for arbitrary $\mathcal{N}$ the emerging spectrum from a clump population containing a wide range of optical depths. Taking uniform spheres for the individual clouds, the required averaging carried out in forming the frequency profile $f(\nu)=1-\left\langle e^{-\tau_{\nu}}\right\rangle$ can first be made over different clump LOS impact parameters (see Appendix B), and then these spatial averages themselves are averaged over the population of clump types with different opacities across their diameters. 9

In Figure 3, panel (a) shows the emerging spectra for different mean numbers $\mathcal{N}$ of clumps per LOS, and panel (b) shows the corresponding local spectral index $\alpha=$ $d \log I_{\nu} / d \log \nu$ versus frequency for each $\mathcal{N}$. The spectra were calculated with the same power-law distribution of clump opacities assumed by IC04, i.e., the $\tau_{0}$ distribution given by

\footnotetext{
9 In the example chosen below with a wide cloud opacity distribution, the average over clump structure has a negligible effect on the final spectrum compared to the effects of averaging over the opacity spectrum.
}

Equation (13) with $\gamma=1.5, \tau_{0, \min }=0.01$, and $\tau_{0, \max }=100 .^{10}$ Given that $f(\nu)<1$ (from its definition), it follows that when $\mathcal{N} \ll 1$, all frequencies obey $\tau_{\mathrm{E}_{\nu}}=\mathcal{N} f(\nu) \ll 1$, in turn implying that $I_{\mathrm{C} \nu} \approx 2 \mathcal{N} k\langle T\rangle \nu^{2} f(\nu) / c^{2}$; that is, the output spectrum has a fixed shape $\nu^{2} f(\nu)$ scaled in intensity by $\mathcal{N}$. This low- $\mathcal{N}$ limit is illustrated by the red curve in Figure 3(a) for the specific case $\mathcal{N}=0.1$; this is the output spectrum found by IC04, and it includes a broad frequency range with intermediate spectral index. This range starts at the frequency at which every cloud in the opacity distribution is optically thick, $\nu_{\min }=\nu_{0} \tau_{0, \min }{ }^{1 / 2.1}$, and ends at the frequency at which all are optically thin, $\nu_{\max }=\nu_{0} \tau_{0, \max }^{1 / 2.1}$. The spectral index $\alpha$ of the $\mathcal{N}<1$ output emission spectrum is therefore intermediate between its optically thick and thin values over the fractional frequency range

$$
\frac{\nu_{\max }}{\nu_{\min }}=\left(\frac{\tau_{0, \text { max }}}{\tau_{0, \text { min }}}\right)^{0.48},
$$

which is about 100 for the ratio $\tau_{0, \max } / \tau_{0, \text { min }}=10^{4}$ employed here. It is interesting to note that this relative spectral width is independent of the absolute values of the optical depth boundaries; changing the lower limit of the $\tau_{0}$ distribution while keeping $\tau_{0, \max } / \tau_{0, \min }$ fixed will only slide this intermediate region toward higher or lower frequencies without changing the $\nu_{\max } / \nu_{\min }$ ratio. Although all spectral indices between 2 and -0.1 occur in this intermediate range (see Figure 3(b)), there exists a "plateau" region where $\alpha$ changes relatively slowly. Integration over the $\tau_{0}$ distribution (Equation (13)) shows that within this plateau region, to leading order, $f(\nu)$ varies as $\nu^{-2.1(\gamma-1)}$ so that the spectral index there is

$$
\alpha \simeq 2-2.1(\gamma-1)
$$

The choice of $\gamma=1.5$ therefore gives $\alpha \approx 0.95$.

Universality of the spectral shape holds only so long as $\mathcal{N}<1$. At larger cloud numbers, the spectral shape becomes dependent on $\mathcal{N}$, with the low-frequency $\nu^{2}$ behavior approaching the high-frequency $\nu^{-0.1}$ domain as $\mathcal{N}$ increases. From Equation (15), the $\nu^{2}$ behavior occurs whenever $\tau_{\mathrm{E}_{\nu}}=\mathcal{N} f(\nu)>1$, which for $\mathcal{N}>1$ is a condition that holds up to the frequency at which $f(\nu)>1 / \mathcal{N}$ is still valid. Given that in the intermediate domain, $f(\nu) \propto\left(\nu_{\min } / \nu\right)^{-2.1(\gamma-1)}$, this means that the lower boundary of the intermediate spectral index range increases from $\nu_{\min }$ to $\nu_{\min } \mathcal{N}^{1 / 2.1(\gamma-1)}$, i.e., $\nu_{\text {min }} \mathcal{N}^{0.95}$ when $\gamma=1.5$. Consistent with this predicted behavior, Figure 3(a) shows that as $\mathcal{N}$ increases to become $\gg 1$, the ensemble spectra saturate against the $\nu^{2}$ asymptote at increasingly higher frequencies, a consequence of the fact that as $\mathcal{N}$ increases, the population has $\tau_{\mathrm{E}_{\nu}}=1$ up to higher frequencies. If we now consider the frequency region at which the transition to an optically thin overall spectral index $\alpha=-0.1$ occurs, then for all the values of $\mathcal{N}$ shown in Figure 3(a), this occurs at a frequency much larger than the one at which $\tau_{\mathrm{E}_{\nu}}=1$ and hence $\tau_{\mathrm{E}_{\nu}} \ll 1$ at this upper turnover. It follows from Equation (15) that around the upper turnover, the ensemble spectrum remains proportional to $\nu^{2} f(\nu)$ scaled by $\mathcal{N}$ even when $\mathcal{N} \gg 1$. The upper frequency of the intermediate spectral index region therefore still occurs at the upper turnover

\footnotetext{
${ }^{10}$ The effects of varying all parameters other than $\mathcal{N}$ have been studied extensively in IC04.
} 
frequency of $\nu^{2} f(\nu)$, i.e., at the frequency in which all clouds in the opacity distribution become optically thin at $\nu_{\max }=$ $\nu_{0} \tau_{0, \max }^{1 / 2.1}$, independent of $\mathcal{N}$. As a consequence of the different dependence on $\mathcal{N}$ of the lower and upper limits of the intermediate spectral index range, the fractional frequency range showing the intermediate spectral index shrinks with $\mathcal{N}$ when $\mathcal{N}>1$. Specifically, when $\gamma=1.5$, the fractional frequency range showing the intermediate spectral index approximates to $\mathcal{N}^{-0.95}\left(\tau_{0, \max } / \tau_{0, \text { min }}\right)^{0.48}$; this also explains the steepening of the spectral index transition region with increasing $\mathcal{N}$, evident in Figure 3(b).

Our results show that Ignace \& Churchwell (2004) correctly identified the unique set of conditions in which clumpy H II regions with a power-law $\tau_{0}^{-\gamma}$ distribution of cloud optical depths can produce the observed $\alpha \simeq 1$ spectra. When $\mathcal{N}<1$, the relative width of the intermediate spectral index region can be substantial when the clump $\tau_{0}$ distribution covers a large range (Equation (16)). The observed spectral index in this intermediate-frequency range is controlled by $\gamma$, with $\gamma=1.5$ giving $\alpha \simeq 1$ (Equation (17)). Our formalism shows for the first time how the output spectrum is affected when $\mathcal{N}>1$, demonstrating that for such cases, the frequency range showing an intermediate spectral index narrows significantly with increasing $\mathcal{N}$.

\subsection{CO Spectral Line Profiles}

Consider line emission from a population of small clouds within a telescope beam coming from an external galaxy or a galactic star-forming region. The resulting output spectrum is the average of the emission over many LOSs and therefore equals the LOS expectation value of spectral line emission (see Section 2.4). As an example of line emission from a clumpy medium, Figure 4 shows model CO spectra for ensembles of identical uniform spherical clouds. ${ }^{11}$ The distribution of cloud LOS velocities is taken as Gaussian with width $\Delta u$, assumed to be much larger than $\Delta v$, the velocity width of each cloud (as defined in Section 2.3). The expected spectrum in such a case is given by Equation (11), which shows that it depends on the product of the total optical depth $\tau_{\mathrm{T}}(v)$ and the clumping factor $K_{\text {line }}$, determined by the mean properties of clouds in the distribution (Equation (10)). Figure 4 shows predicted ${ }^{12} \mathrm{CO}(1-0)$ and ${ }^{13} \mathrm{CO}(1-0)$ spectra, with a ${ }^{13} \mathrm{CO}(1-0)$ absorption coefficient that is $1 / 60$ that of ${ }^{12} \mathrm{CO}(1-0)$. Different columns show spectra for different values of the total ${ }^{12} \mathrm{CO}(1-0)$ optical depth at zero velocity, $\tau_{\mathrm{T}}^{12}(0)$, as listed at the bottom of each column. Different rows present different values of the mean single cloud $\left\langle\tau_{0}^{12}\right\rangle$, the line-center optical depth averaged over the face of a cloud; the corresponding $K_{\text {line }}$ is listed to the right of each row. Since $\tau_{\mathrm{T}}^{12}(0)=N(0)\left\langle\tau_{0}^{12}\right\rangle \Delta v$, for each panel's combination of $\tau_{\mathrm{T}}^{12}(0)$ and $\left\langle\tau_{0}^{12}\right\rangle$, there is a corresponding value of $N(0) \Delta v$, the number of clumps per LOS with a center velocity within $\pm \Delta v / 2$ of zero velocity, as given in the top left corner of each panel.

To understand the displayed profiles, it helps to recall that the brightness temperature of a smooth-density source with constant excitation temperature is $T_{\mathrm{b}}=T_{\mathrm{x}}[1-\exp (-\tau(v))]$. Therefore, when the line-center optical depth obeys $\tau(0)<1$,

\footnotetext{
11 As noted in Section 3, the exact cloud shape has very little effect on the emerging spectra from a cloud population; for identical clouds, any cloud shape that has the same mean opacity averaged over its face at cloud central velocity will have almost identical spectra.
}

the emission is proportional to $\tau(v)$, displaying the Gaussian velocity distribution profile. In contrast, when $\tau(0)>1$, the emission develops a flat-top profile that broadens with increasing $\tau(0)$. This same familiar behavior is expected in a clumpy source when individual clouds are optically thin, since clumping has no effect in that case (Section 2), with the profile dominated by the cloud ensemble Gaussian motions, since we assume $\Delta u \gg \Delta v$. That is indeed what Figure 4 shows. In all the panels, individual clouds are optically thin in the ${ }^{13} \mathrm{CO}(1-0)$ transition because of the ${ }^{13} \mathrm{CO}$ small abundance. As a result, the ${ }^{13} \mathrm{CO}(1-0)$ line profiles are identical within the panels of each column, for which total optical depth is the same, and every row shows the same familiar evolution for an optically thin line profile-Gaussian with peak intensity proportional to the linecenter optical depth. In the bottom two rows, individual clouds are also optically thin in ${ }^{12} \mathrm{CO}(1-0)$, so they in turn display the same expected profile development with increasing total optical depth to a fully saturated flat top in the rightmost panels. However, when individual clouds become optically thick, clumping induces profile behavior completely unexpected from the smooth-density experience. This unique behavior stands out when the panels are followed up the figure's rightmost column, where $\tau_{\mathrm{T}}{ }^{12}(0)=10$ : the flat top gets narrower as the optical depth per cloud increases, until finally the original Gaussian profile emerges almost intact when the line-center optical depth of a cloud is $\tau_{0}^{12}=10$. While it is impossible in the case of a smooth-density gas that such a high $\tau_{\mathrm{T}}$ could have a velocity profile close to Gaussian, this can occur as a natural consequence of clumpiness. Since the effective optical depth is $\tau_{\mathrm{E}}=K_{\text {line }} \tau_{\mathrm{T}}$ (Equation (10)), the line becomes effectively thin when the optical depth of individual clouds is sufficiently high, a result of the decline of $K_{\text {line }}$ (see Figure 2; note also the value of $K_{\text {line }}$ listed to the right of each row). While in the top right panel of Figure 4, the overall optical depth $\tau_{\mathrm{T}}$ is high, on average, it is concentrated into only one very optically thick cloud. Since the probability distribution of clouds per LOS is a Poisson distribution, the fraction $e^{-1}(\sim 40 \%)$ of all sightlines does not intercept any cloud. With only a fraction of the surface area contributing to the observed radiation, the source brightness temperature is less than the cloud excitation temperature, and the line remains unsaturated even at its center. At higher and lower velocities, the covering factor of the optically thick clouds decreases, so the resulting spectral profile closely follows the Gaussian profile of the number of clouds per cloud LOS velocity width.

The richness of line spectra from clumpy media, indicated by the systematic overview in Figure 4 of profile variation with clump and total optical depth, has important consequences for spectral studies of external galaxies. It is important to note that although the calculations behind this figure assume the specific case of identical clouds, the resulting spectral profiles are in fact universally applicable to a medium containing any distribution of different cloud types. Equation (11) shows that clumpy medium emission is fully specified by $\tau_{\mathrm{T}}$ and the clumping factor $K_{\text {line }}$, which is listed on the right of each row in the figure. A remarkable result is that any medium containing a mixture of nonidentical clouds with the same value of $K_{\text {line }}$ as one of the rows of Figure 4 will have identical spectral shapes as a function of $\tau_{\mathrm{T}}{ }^{12}(0)$, the total optical depth at profile center.

\subsubsection{Clumping Effects on Spectral Line Ratios}

Measurements of intensity ratios between spectral lines provide a powerful tool to constrain chemical and physical 


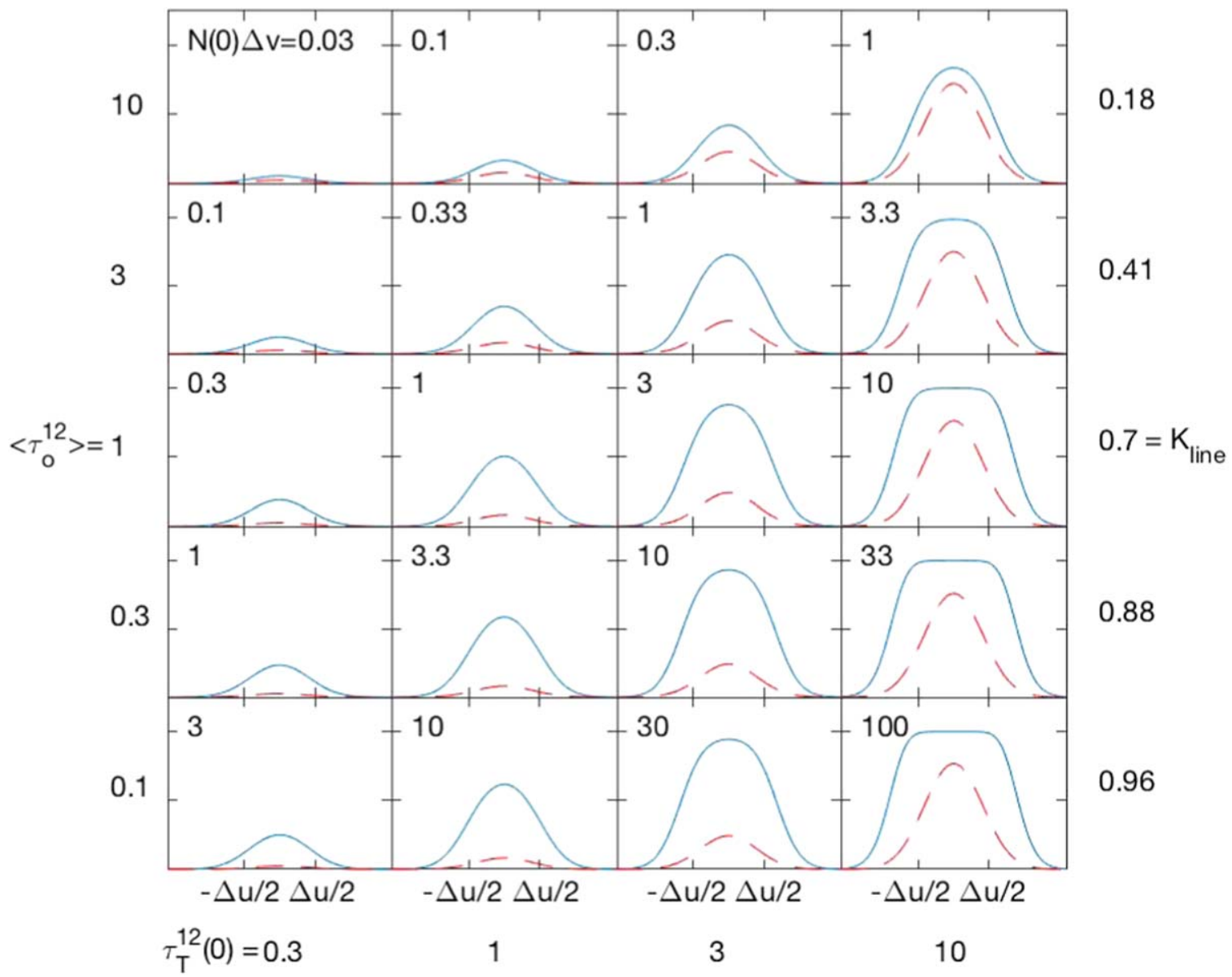

Figure 4. Spectral line emission from an ensemble of clouds whose Gaussian LOS velocity distribution width is $\Delta u \gg \Delta v$, the internal velocity width of each cloud. Shown is a sample of CO spectral profiles for different combinations of individual cloud optical depths (rows) and total optical depths (columns). Each panel shows plots of brightness temperature vs. velocity for ${ }^{12} \mathrm{CO}(1-0)$ (solid blue lines) and ${ }^{13} \mathrm{CO}(1-0)$ (dashed red lines, multiplied by 5 to become more visible). Each $x$-axis is centered on the line center, with tick marks shown at $\pm \frac{1}{2} \Delta u$. Along each $y$-axis, the top tick is placed at the ${ }^{12} \mathrm{CO}$ line-center brightness temperature of the bottom right panel. Rows are labeled on the left side by $\left\langle\tau_{0}^{12}\right\rangle$, the single-cloud line-center ${ }^{12} \mathrm{CO}$ optical depth averaged over the cloud face, and on the right side by the corresponding line clumping factor $K_{\text {line }}$ (Equation (10)). Columns are labeled at the bottom by $\tau_{\mathrm{T}}^{12}(0)$, the total ${ }^{12} \mathrm{CO}$ optical depth at line center (Equation (9)). For $\tau_{\mathrm{T}}^{12}(0)$ to be the same for all the panels in each column, the number of clouds intercepted, on average, along the LOS within $\pm \frac{1}{2} \Delta v$ from line center must vary; this value is indicated in the top left corner of every panel. For more details and interpretation, see the text (Section 4.2).

conditions in astrophysical gases. Such ratios depend on physical parameters such as the relative abundance of molecular species and the excitation of the two transition lines. Often, models that fit line ratios assume uniform slabs of gas and neglect the potential effects of clumping on small scales within these slabs. However, if these small-scale clumps do exist and are optically thick, then clumping can significantly impact the observed line ratios; without accounting for such effects, erroneous physical conditions can be deduced. Equation (11) gives the general expression for the observed line profile in the case of clumping, with the profile intensity and shape depending on the brightness temperature of the line, the total optical depth through the gas, and the clumping factor, $K_{\text {line }}$; the latter, in turn, depends on the optical depth of individual clouds (see Equation (10)).

Line ratios can be calculated by employing either brightness profiles integrated over all velocities of the two transitions or peak brightness temperatures at the centers of the two profiles. Whichever procedure is used, there are in general four separate opacity-related quantities that affect an observed line ratio, comprising the total and single-cloud optical depths for each of the two transitions. The line profiles shown in Figure 4 illustrate a two-dimensional subspace of this general case in which the total and single-cloud optical depths of the weaker line (here ${ }^{13} \mathrm{CO}(1-0)$ ) are set at $\ll 1$, while for the stronger line $\left({ }^{12} \mathrm{CO}(1-0)\right)$, these two quantities both vary from $\ll 1$ to $\gg 1$.

The four panels in the bottom left corner of Figure 4 illustrate the situation when both total and single-cloud optical depths of ${ }^{12} \mathrm{CO}(1-0)$ are $\ll 1$, in which case there are no opacity effects. In this regime, the observed line ratio equals the intrinsic ratio of line strengths determined by the gas physical conditions; for the displayed pair, this is largely determined by the ${ }^{12} \mathrm{CO}:{ }^{13} \mathrm{CO}$ abundance ratio. The four panels in the bottom right corner have total $\tau_{\mathrm{T}} \gg 1$, but individual clouds have $\tau \ll 1$. In this region, the ${ }^{12} \mathrm{CO}(1-0)$ line profile saturates at line center, reducing the observed line ratio. In principle, the saturation of the brighter line can be detected from the relative line shapes of the two transitions, so opacity effects on the line ratio can be corrected for. In practice, though, such corrections require high signal-to-noise observations that are often not available. 
The case of low total but high single-cloud optical depth is shown in the four top left panels of Figure 4. In these spectra, the high single-cloud opacity causes clumping factors $K_{\text {line }}<1$, reducing the ${ }^{12} \mathrm{CO}(1-0)$ line effective optical depth (Equation (11)) and hence its intensity, thus reducing the line

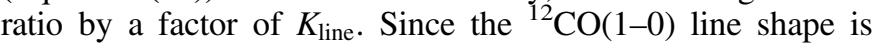
unaffected by having optically thick clouds, it is in principle impossible to determine whether a reduction in observed line ratio reflects clump opacity effects or an intrinsically low line ratio. The final regime is illustrated by the figure's four top right panels, where both single-cloud and total optical depth are simultaneously high. Here the effects of both the reduction of effective opacity and the saturation of the ${ }^{12} \mathrm{CO}$ line profile are in operation; their joint effect can be determined from Equation (11).

In the above, we have carefully defined and distinguished the independent quantities of total and single-cloud optical depth. Figure 4 illustrates that if either of these optical depths is $>1$ in either one of the transitions, then the observed line ratio will be significantly different from its intrinsic value. Since high optical depths, either single-cloud or total, often induce only small or imperceptible changes in line shapes, it is usually not possible to detect their effect observationally. The best that can be done is if a given transition is suspected (either a priori or based on other line ratios) to have a high single-cloud or total optical depth, then line ratios involving that transition should not be used in fitting for gas physical conditions; such a transition could reliably be used only to set limits on line ratios, which could then be exploited in physical modeling.

\section{Discussion}

This paper brings to completion the Natta \& Panagia (1984) approach to radiative transfer in clumpy media, showing that such media can be reliably modeled as collections of structureless clouds ("mega-particles") characterized by a single property: optical depth. The actual clouds can have a wide range of properties, including different geometrical shapes, opacities, emissivities, spectral shapes, bulk velocities, internal structures, and orientations, all of which can vary along the LOS. With proper averaging, all of these properties can be rigorously encapsulated in an ensemble of identical clouds, and, to a good degree of approximation, the geometry of these average clouds is irrelevant. ${ }^{12}$

The simplicity of the formalism presented here has enabled us to readily calculate clumpy emission spectra for a number of current problems, including ensembles of UCHIIs (Section 4.1), CO spectral lines (Section 4.2), and synchrotron emission accompanied by free-free absorption from supernovae and compact starbursts in ultraluminous IR galaxies (Appendix C). Our results replicate and extend numerous earlier studies. We show that Ignace \& Churchwell (2004) correctly identified the unique set of conditions in which clumpy H II regions with a power-law distribution of cloud optical depths can produce $\alpha \simeq 1$ spectra (Section 4.1). And, in the case of spectral line observations, we show that it is impossible, even in principle, to distinguish the effect of atomic and molecular abundances on line ratios from the clumping effects of optically thick clouds (Section 4.2.1).

\footnotetext{
${ }^{12}$ It is interesting to note the similarity with approaches taken in the context of radiation propagation in porous media (see Taine et al. 2008).
}

The effective optical depth of a clumpy medium (Equation (1)) arises from the result of our formalism for $\left\langle e^{-\tau_{\nu}}\right\rangle$, the first moment of the transmission factor distribution. Higher moments can be calculated just as easily - the $m$ th moment, $e^{-m \tau_{\nu}}$, distribution average is simply $\exp \left[-\mathcal{N}\left(1-\left\langle e^{-m \tau_{\nu}}\right\rangle\right)\right]$, as directly obtained from the derivations in Appendix A.2. Such moments can yield useful information about the cloud distribution. Tauber (1996) pointed out that a possible route to explore clumpiness is to observe emission lines with very high spectral resolution and signal-tonoise ratios, analyze the fluctuations in brightness temperature present on the line shape, and infer from them the properties of the clumps present in the beam. Assuming identical clumps and employing the Martin et al. (1984) model, Tauber (1996) computed the expected fluctuations for a wide range of clump optical depths. Other studies of the fluctuation effect, both earlier (Tauber et al. 1991) and later (Pirogov et al. 2012), were restricted to optically thin clumps. Based on our clump formalism, it is straightforward to show that the transmission factor variance obeys

$$
\begin{aligned}
& \left\langle\left(e^{-\tau_{\nu}}-\left\langle e^{-\tau_{\nu}}\right\rangle\right)^{2}\right\rangle \\
& =\exp \left[-\mathcal{N}\left(1-\left\langle e^{-2 \tau_{\nu}}\right\rangle\right)\right]-\exp \left[-2 \mathcal{N}\left(1-\left\langle e^{-\tau_{\nu}}\right\rangle\right)\right]
\end{aligned}
$$

This simple expression is completely general and encompasses the results of all previous studies. It enables analysis of spectral variance, as performed by Tauber et al. (1991) and Tauber (1996), for arbitrary sources without any model restrictions. Such analysis can directly yield $\mathcal{N}$, the total number of clouds along the LOS. A similar utility exists for higher moments, which can be derived just as easily.

While the formalism developed here is quite general, it does rest on some fundamental assumptions. The clump volume filling factor is assumed to be small enough that departures from Poisson statistics are small. In practice, Monte Carlo simulations (see Section 2.1) show the formalism to give good estimates up to quite large volume filling factors $(\sim 10 \%)$. When larger filling factors are desired, modeling would have to rely on Monte Carlo simulations. Next, the absorption and emission properties of single clouds are assumed to be unaffected by the radiation generated by the clumpy medium. Relaxing this assumption requires an iterative procedure that starts with initial cloud properties, such as level populations or dust temperature, determined in the absence of cloud emission. In subsequent steps, the clump formalism is used to calculate the expected radiation field, which is then added to the calculation of individual cloud properties and reiterated until convergence.

Finally, the formalism assumes random placement in space of individual clouds, such that the presence of another cloud nearby neither increases nor decreases the probability for a cloud at a given position. However, there is evidence suggesting that galactic clouds could be fractal (Falgarone et al. 1991; Elmegreen \& Scalo 2004), with small high-density clumps embedded within larger lower-density clouds. Nevertheless, there are reasons to expect our formalism to give approximately correct answers even in such a case. Consider a critical size scale at which the optical depth is approximately unity, such that smaller, denser clumps are optically thick but larger ones are optically thin. Then the fact that the latter are correlated in position with clumps of the critical size has little effect. Smaller, very optically thick cores will be embedded 
within the already optically thick critical-scale clouds; thus, their contributions to the total spectrum will be small. Applying our formalism and considering only the clouds at the critical size and larger should therefore produce reasonably accurate results. In future work, we hope to quantify the formalism accuracy when applied to fractal clouds.

We are grateful to the anonymous referee for useful comments. ME acknowledges the help of Frank Heymann and Robert Nikutta.

\section{Appendix A \\ Analytic Results for Small Volume Filling Factors}

\section{A.1. Cloud Distributions}

The fundamental function describing the distribution of clouds with velocity vector $\boldsymbol{u}$ at position $\boldsymbol{r}$ is $n(\boldsymbol{r}, \boldsymbol{u})$, the number of clouds per unit volumes of space and velocity space. When interacting with continuum radiation, the cloud velocity is irrelevant and can be integrated out to produce $n(\boldsymbol{r})=\int n(\boldsymbol{r}, \boldsymbol{u}) d^{3} \boldsymbol{u}$, the number of clouds per unit volume at position $\boldsymbol{r}$. For radiation propagating along some path, denote by $A$ the cloud area perpendicular to the path. Then the number of clouds a photon encounters per unit length is $N=n A$, the inverse of the local mean free path. As shown by Nenkova et al. (2002, 2008), the distribution $N$ suffices to describe the clumpy radiative transfer problem when the volume filling factor is small; the volume density $n$ and the cloud area $A$ do not enter separately in that case. Then the overall number of clouds a photon encounters, on average, between any points $s_{1}$ and $s_{2}$ along the path is

$$
\mathcal{N}\left(s_{1}, s_{2}\right)=\int_{s_{1}}^{s_{2}} N(s) d s
$$

Generalizing to an arbitrary mix of cloud types is as simple as adding independent variables, one for every additional cloud property; introducing the distribution $N$; and deriving the corresponding $\mathcal{N}$ for each type separately.

In the case of line radiation, one needs to identify the clouds with a particular LOS velocity $u$. To that end, integrate $n(\boldsymbol{r}, \boldsymbol{u})$ over the velocity components perpendicular to the path to get $n(\boldsymbol{r}, u)=\int n(\boldsymbol{r}, \boldsymbol{u}) d^{2} u_{\perp}$, the cloud number density per unit volume and unit LOS velocity $u$. As before, $N=n A$ and the number of clouds encountered along the path between $s$ and $s+d s$ with LOS velocity between $u$ and $u+d u$ is $N(u, s) d s d u$. Then the total number of clouds encountered between $s$ and $s+d s$ at all velocities is $N(s) d s$, while the total number encountered along the entire path with velocities between $u$ and $u+d u$ is $N(u) d u$, where

$$
N(s)=\int N(u, s) d u, \quad N(u)=\int N(u, s) d s .
$$

The total number of clouds along the entire path and at all LOS velocities is

$$
\mathcal{N}=\int N(s) d s=\int N(u) d u
$$

\section{A.2. Absorption in Clumpy Media}

Consider the absorption of background radiation by a foreground ensemble of identical clouds. Denote by $e^{-\tau_{\nu}}$ the transmission through a single cloud at frequency $\nu$ and assume that interaction with the radiation does not change $\tau_{\nu}$. Let $\mathcal{N}$ be the mean number of clouds along a given LOS. With the assumption that the clouds are placed randomly and their volume filling factor is small, Natta \& Panagia (1984) employed Poisson statistics to show that the mean transmission factor averaged over many realizations of that LOS is $\left\langle e^{-\tau_{\nu}}\right\rangle=\exp \left(-\tau_{\mathrm{E}_{\nu}}\right)$, where

$$
\tau_{\mathrm{E}_{\nu}}=\mathcal{N}\left(1-e^{-\tau_{\nu}}\right)
$$

is the effective optical depth of the clumpy medium. Consider now a mixture of $n$ types of clouds with each type having optical depth $\tau_{\nu, i}(i=1,2 \ldots n)$ and comprising a fraction $f_{i}=\mathcal{N}_{i} / \mathcal{N}$ of the average total number of clouds along the $\operatorname{LOS}\left(\sum f_{i}=1\right)$. The probability of encountering any given combination of number of clouds of each type is given by a multivariate Poisson probability distribution. Repeating the Natta \& Panagia averaging procedure over all possible cloud types, straightforward algebraic manipulations show that the effective optical depth is now

$$
\tau_{\mathrm{E}_{\nu}}=\mathcal{N}\left(1-\left\langle e^{-\tau_{\nu}}\right\rangle\right)
$$

(cf. Equation (1)), where

$$
\left\langle e^{-\tau_{\nu}}\right\rangle=\sum_{i} f_{i} e^{-\tau_{\nu, i}}
$$

is the mean single-cloud transmission factor at frequency $\nu$. In the case that the cloud type varies continuously with some parameter $t$, the discrete fractional abundance $f_{i}$ is replaced by $f(t) d t$ (with $\int f(t) d t=1$ ), the fraction of clouds in the parameter interval $[t, t+d t]$, and the sum is replaced by the integral $\int f(t) e^{-\tau_{\nu}(t)} d t$. Note that the variable $t$ can refer to any label defining cloud type. In the simplest case, it could refer to a single opacity characterizing each cloud, and $f(t)$ would then be the cloud opacity distribution. It could also, however, refer to the impact parameter of the LOS relative to the center of, say, spherically symmetric clouds or an angle describing filamentary cloud orientations (see Appendix B). Furthermore, the cloud average can be made over any number of dimensions of continuous parameters, $t_{1}, t_{2} \ldots$, which describe the cloud population with the appropriate probability distribution $f\left(t_{1}, t_{2} \ldots\right)$.

The above results also follow from an alternative derivation that utilizes a radiative transfer approach. ${ }^{13}$ Start with the case of single-type clouds and introduce $\eta(s)=N(s) / \mathcal{N}$; then the number of clouds encountered in the differential segment $d s$ along the radiation path is $N(s)=\mathcal{N} \eta(s) d s$ (note that $\left.\int \eta d s=1\right)$. Since each cloud absorbs the fractional amount $1-e^{-\tau_{\nu}}$ of impinging radiation, in traversing $d s$, the intensity is attenuated by the amount $d I_{\nu}=-I_{\nu} \mathcal{N}\left(1-e^{-\tau_{\nu}}\right) \eta d s$. Integrating along the full path yields the Natta \& Panagia result (Equation (22)). Extending this approach to a cloud mixture is straightforward. Introduce $\eta_{i}(s)=N_{i}(s) / \mathcal{N}_{i}$, the spatial distribution profile of type- $i$ clouds $\left(\int \eta_{i} d s=1\right)$; then the number of such clouds encountered in the differential segment $d s$ is $N_{i}(s)=\mathcal{N} f_{i} \eta_{i}(s) d s$. Since each cloud absorbs the fraction $1-e^{-\tau_{\nu, i}}$ of propagating radiation, radiative transfer in

\footnotetext{
13 A somewhat similar approach has been used by Lacki (2013).
} 
clumpy media is controlled by

$$
\frac{d I_{\nu}}{I_{\nu}}=-\mathcal{N} \sum_{i} f_{i}\left(1-e^{-\tau_{\nu, i}}\right) \eta_{i}(s) d s .
$$

Integrating along the full path and summing over all cloud types leads directly to $\tau_{\mathrm{E}_{\nu}}$ from Equations (23) and (24).

\section{A.3. Emission from Clumpy Media}

The fundamental expression for continuum emission from single-type clouds has been given in Nenkova et al. (2002, 2008). To calculate the emission along a given LOS in this case, denote by $S_{\mathrm{C} \nu}(s)$ the single-cloud source function at position $s$; this is the increase in brightness of radiation propagating along the LOS because of the emission from a single cloud. With $N(s)$, the expected number of clouds per unit length, the overall number of clouds in the differential segment $d s$ along the path is $N(s) d s$, and the intensity generated in that segment is $S_{\mathrm{C} \nu}(s) N(s) d s$. This is the input radiation to the rest of the path, which contains $\mathcal{N}(s)=\int_{s} N\left(s^{\prime}\right) d s^{\prime}$ clouds, on average. Therefore, the mean transmission factor for that remaining segment is $\exp \left[-\tau_{\mathrm{E}_{\nu}}(s)\right]$, where $\tau_{\mathrm{E}_{\nu}}(s)=$ $\mathcal{N}(s)\left(1-e^{-\tau_{\nu}}\right)$, and the emerging intensity is

$$
I_{\mathrm{C} \nu}=\int e^{-\tau_{E \nu}(s)} S_{\mathrm{C} \nu}(s) N(s) d s .
$$

Generalizing to a cloud distribution is straightforward. There are $N_{i}(s)$ clouds of type $i$ per unit length, on average, each with a source function $S_{\mathrm{C} \nu_{i}}(s)$. Then the intensity generated per unit length is $\sum_{i} N_{i} S_{\mathrm{C} \nu_{i}}(s)=N\left\langle S_{\mathrm{C} \nu}\right\rangle$, where

$$
\left\langle S_{\mathrm{C} \nu}\right\rangle=\sum_{i} f_{i} S_{\mathrm{C} \nu_{i}}
$$

and a similar averaging procedure when the cloud distribution is characterized by a continuous variable. Note that the clouds that dominate the averages in $\langle S\rangle$ and $\left\langle e^{-\tau}\right\rangle$ (Equation (24)) can be different from each other. Because the statistical variations of the emission and foreground absorption are uncorrelated, the mean contribution of element $d s$ to the received intensity equals its mean emission times the mean foreground transmission. This leads directly to the result in Equation (2).

\section{A.4. Spectral Line Absorption}

The optical depth of a line centered on frequency $\nu_{0}$ is $\tau(\nu)=\tau_{0} \Phi\left(\nu-\nu_{0}\right)$, where $\tau_{0}$ is the line-center optical depth and $\Phi$ is the line profile normalized to $\Phi(0)=1$. The line width can be defined from $\Delta \nu \equiv \int \Phi\left(\nu-\nu_{0}\right) d \nu$. In addition to the potential intrinsic variation of $\tau_{0}$ and $\Delta \nu$ from cloud to cloud, $\nu_{0}$ can also vary because of cloud motions. The line-center frequency of a cloud with LOS velocity $u$ is shifted to $\nu_{0}(1-u / c)$, and its optical depth becomes

$$
\tau(\nu, u)=\tau_{0} \Phi\left[\nu-\nu_{0}(1-u / c)\right] .
$$

Based on the approach described in Appendix A.2 above, the effective line opacity at frequency $\nu$ of an ensemble of clouds is given by Equation (1) with the quantity $\left\langle e^{-\tau_{\nu}}\right\rangle$ now including an average over different cloud-center velocities. Since we wish to handle the cloud velocity distribution separately from all other possible variations of cloud properties (i.e., $\tau_{0}$ and $\Delta \nu$ ), we lump them together into a single symbolic variable $t$. Then $\left\langle e^{-\tau_{\nu}}\right\rangle=\iint f(u, t) e^{-\tau(\nu, u)} d u d t$, where the distribution $f$ describes the fractional number of clouds with LOS velocity $u$ and value $t$ for the other cloud properties.

If the fraction of clouds of different types $t$ is independent of velocity, then $f(u, t)=g(t) N(u) / \mathcal{N}$, where $\mathcal{N}$ is the overall number of clouds encountered, on average, along the path; $N(u)$ is the number per unit LOS bandwidth $u$ (see Appendix A.1 above); and $g(t)$ is the probability distribution of all other cloud properties $\left(\int g(t) d t=1\right)$. Then Equation (1) for the line effective optical depth becomes

$$
\tau_{\mathrm{E}_{\nu}}=\int N(u)\left(1-\left\langle e^{-\tau(\nu, u)}\right\rangle\right) d u,
$$

where $\left\langle e^{-\tau(\nu, u)}\right\rangle=\int g(t) e^{-\tau(\nu, u)} d t$ is the cloud mean transmission factor, averaged over all cloud properties other than LOS velocity. Instead of frequency $\nu$, the equivalent Doppler velocity $v=c\left(1-\nu / \nu_{0}\right)$ is customarily employed. Then the optical depth becomes $\tau(u, v)=\tau_{0} \Phi(u-v)$, and the profile width is $\Delta v=c \Delta \nu / \nu_{0}$, a velocity scale that characterizes the cloud internal motions. Replacing frequency $\nu$ with equivalent Doppler velocity $v$ in Equation (29) yields the result in Equation (7).

\section{A.5. Spectral Line Emission}

Start with a population of identical clouds, described by the distribution $N(s, u)$ (Appendix A.1). Denote by $S_{0}(s)$ the linecenter brightness of a cloud at rest at position $s$; then the cloud emission at frequency $\nu$ is $S_{0} \psi\left(\nu-\nu_{0}\right)$. When the cloud is optically thin, $\psi=\Phi$, the emission profile simply has the line (Doppler) shape. In the general case, both $S_{0}$ and $\psi(\nu)$ are determined only after a detailed solution of the radiative transfer problem for single clouds. When the cloud is moving with Doppler velocity $u$, its emission at frequency $\nu$ becomes $S_{0}(s) \psi\left[\nu-\nu_{0}(1-u / c)\right]$; thus, the average emission at frequency $\nu$ from the cloud ensemble at position $s$ is described by the source function

$$
\begin{aligned}
S_{\mathrm{C} \nu}(s) & =S_{0}(s) \Psi_{\nu} \\
\text { where } \quad \Psi_{\nu} & =\frac{1}{N(s)} \int \psi\left[\nu-\nu_{0}(1-u / c)\right] N(s, u) d u .
\end{aligned}
$$

The intensity generated in segment $d s$ at frequency $\nu$ is $S_{\mathrm{C} \nu}(s) N(s) d s$, and, while traveling through the rest of the path, it is attenuated by $\exp \left[-\tau_{\mathrm{E}_{\nu}}(s)\right]$, where $\tau_{\mathrm{E}_{\nu}}(s)$, the line effective optical depth from point $s$, is calculated from Equation (29). Therefore, in complete analogy with the continuum case (Equation (2)), the emerging line intensity is

$$
I_{\mathrm{C} \nu}=\int e^{-\tau_{E \nu}(s)} S_{\mathrm{C} \nu}(s) N(s) d s .
$$

As before, generalizing this result to a mix of clouds is straightforward. Adding an index $i$ to differentiate between cloud species and denoting by $\Psi_{i \nu}$ the emission profile of the $i$ th species as defined in Equation (30), the brightness generated per unit length is

$$
\begin{aligned}
& \sum_{i} S_{0 i}(s) \Psi_{i \nu} N_{i}(s)=N(s) \sum_{i} f_{i}(s) S_{\mathrm{C} \nu_{i}}(s)(s) \\
& \quad=N(s)\left\langle S_{\mathrm{C} \nu}(s)\right\rangle .
\end{aligned}
$$


When the cloud mix is described by a continuous parameter, the sums in this relation are trivially replaced by integrals. From the last two relations, it follows immediately that the line intensity emerging from a clumpy medium is

$$
I_{\mathrm{C} \nu}=\int e^{-\tau_{E \nu}(s)}\left\langle S_{\mathrm{C} \nu}(s)\right\rangle N(s) d s,
$$

the same fundamental expression as for the continuum case (Equation (2)).

Inside a cloud, the source function is $B_{\nu}\left(T_{\mathrm{x}}\right)$, where $B_{\nu}$ is the Planck function and $T_{\mathrm{x}}$ is the local line excitation temperature (e.g., Elitzur 1992). A widely used approximation is to assume a constant $T_{\mathrm{x}}$, neglecting its variation with position inside the cloud. Then the emission intensity of a single cloud at rest, the source function of the clumpy medium, is $S_{\nu}=B_{\nu_{0}}\left(T_{\mathrm{x}}\right)\left(1-e^{-\tau_{\nu}}\right)$. When the velocity distribution is independent of position along the LOS, this implies $\Psi_{\nu}=\left\langle 1-e^{-\tau_{\nu}}\right\rangle$, as well as $\tau_{\mathrm{E}_{\nu}}=$ $\mathcal{N}(s)\left\langle 1-e^{-\tau_{\nu}}\right\rangle$. Expressing intensity in terms of equivalent brightness temperature $T_{\mathrm{b}}$ and assuming both $T_{\mathrm{b}}$ and $T_{\mathrm{x}}$ to be in the Rayleigh-Jeans domain, Equation (33) yields

$$
T_{\mathrm{b}_{\nu}}=\left\langle T_{\mathrm{x}}\right\rangle\left(1-\left\langle e^{-\tau_{E \nu}}\right\rangle\right),
$$

where we have additionally assumed that the locally averaged excitation temperature $\left\langle T_{\mathrm{x}}\right\rangle$ is the same everywhere in the clumpy region.

\section{Appendix B}

\section{Averages for Spheres, Slabs, and Filaments}

The clumping correction factor involves the averages $\langle\tau\rangle$ and $\left\langle e^{-\tau}\right\rangle$ over all cloud orientations (Equation (6)). In the case of uniform clouds, these averages become straight geometrical integrations, which we now consider for spherical and filamentary clouds. Additionally, thanks to the symmetry properties of the planar geometry, the slab radiative transfer problem is altogether independent of the density profile and is considered here too. The frequency index is omitted, since only the geometrical averaging is considered here.

A uniform spherical cloud is fully characterized by its radius $R$ and optical depth across the diameter $\tau_{1}$; the optical depth across a chord with length $\ell$ is $\tau_{1} \ell / 2 R$. The path length along impact parameter $b$ is $\ell(b)=2 \sqrt{R^{2}-b^{2}}$. Averaging over all impact parameters yields

$$
\begin{aligned}
\langle\tau\rangle & =2 \tau_{1} \int_{0}^{1} \sqrt{1-\rho^{2}} \rho d \rho=\frac{2}{3} \tau_{1}, \\
\left\langle e^{-\tau}\right\rangle & =2 \int_{0}^{1} \exp \left(-\tau_{1} \sqrt{1-\rho^{2}}\right) \rho d \rho \\
& =\frac{2}{\tau_{1}^{2}}\left[1-\left(1+\tau_{1}\right) e^{-\tau_{1}}\right] .
\end{aligned}
$$

The analytic result for $\left\langle e^{-\tau}\right\rangle$ for uniform spheres was noted previously by Ignace $\&$ Churchwell (2004).

Consider now a filament with radius $R$ and optical depth $\tau_{1}$ across the diameter. The optical depth along a path displaced by distance $y$ and inclined by angle $\theta$ from the axis (Figure 5) is

$$
\tau(y, \theta)=\frac{\tau_{1}}{\sin \theta} \sqrt{1-\rho^{2}}, \quad \text { where } \quad \rho=\frac{y}{R} .
$$

Significantly, $\tau$ does not depend on the filament's length; it depends only on radius and viewing angle, so long as the path does not intersect either end cap. Assuming a large aspect ratio (length-to-width) so that the contributions of the end caps can be neglected, averaging over $y$ and $\theta$ yields

$$
\begin{gathered}
\langle\tau\rangle=\tau_{1} \int_{0}^{1} d \mu \int_{0}^{1} \sqrt{\frac{1-\rho^{2}}{1-\mu^{2}}} d \rho=\frac{\pi^{2}}{8} \tau_{1}, \\
\left\langle e^{-\tau}\right\rangle=\int_{0}^{1} d \mu \int_{0}^{1} \exp \left(-\tau_{1} \sqrt{\frac{1-\rho^{2}}{1-\mu^{2}}}\right) d \rho .
\end{gathered}
$$

The planar geometry, with $\tau_{1}$ the optical depth along the normal, is handled similarly. The path length is now independent of impact parameter while having the same dependence on viewing angle, $\tau(\theta)=\tau_{1} / \sin \theta$, leading to

$$
\begin{gathered}
\langle\tau\rangle=\tau_{1} \int_{0}^{1} \frac{d \mu}{\sqrt{1-\mu^{2}}}=\frac{\pi}{2} \tau_{1}, \\
\left\langle e^{-\tau}\right\rangle=\int_{0}^{1} \exp \left(-\tau_{1} / \sqrt{1-\mu^{2}}\right) d \mu .
\end{gathered}
$$

While the integrals for $\left\langle e^{-\tau}\right\rangle$ cannot be performed analytically for slabs and filaments, the similarity with the spherical case of these integrals explains the similarity of the $K$-factor as a function of $\langle\tau\rangle$ for the three geometries (Figure 2).

It may be worthwhile to note a simple result for the averaging over uniform clouds of arbitrary shape. ${ }^{14}$ With the $z$-axis along the LOS and $x-y$ the plane of the sky, the length through the cloud at point $(x, y)$ is $\ell(x, y)$. Averaging over the observed area yields

$$
\bar{\ell}=\frac{\int \ell(x, y) d x d y}{\int d x d y}=\frac{V}{A_{\perp}},
$$

where $V$ is the volume of the cloud and $A_{\perp}$ is its projected area on the plane of the sky. With an arbitrary cloud shape, we must further average over all cloud orientations. Denote such averaging by \langle\rangle ; then the desired quantity is

$$
\langle\ell\rangle=V\left\langle\frac{1}{A_{\perp}}\right\rangle .
$$

The results for $\langle\tau\rangle$ for both uniform spheres (Equation (35a)) and filaments (Equation (37a)) are readily recovered from this general expression.

\section{Appendix C \\ Synchrotron Emission with Free-free Absorption}

Observations of radio emission from compact starbursts in ultraluminous IR galaxies are commonly fitted by a model first proposed by Condon et al. (1991) that combines the effects of star formation-induced synchrotron emission and free-free absorption. This specific model belongs to a general class of two-component mixtures of uniformly distributed emitters and absorbers; examples include ionized gas mixed with dust (Natta \& Panagia 1984) and a uniform mixture of stars and dust (Thronson et al. 1990). In these admixtures, radiation generated by the emitting component (ionized gas, stars, etc.) is selectively attenuated by the smoothly distributed (i.e., not clumped) absorbing material, which itself does not emit appreciably at the relevant wavelengths. Consider a slab containing such a mixture with $\tau_{\mathrm{T}_{\nu}}$ the total optical depth for

\footnotetext{
${ }^{14}$ This approach was first noted by Frank Heymann.
} 


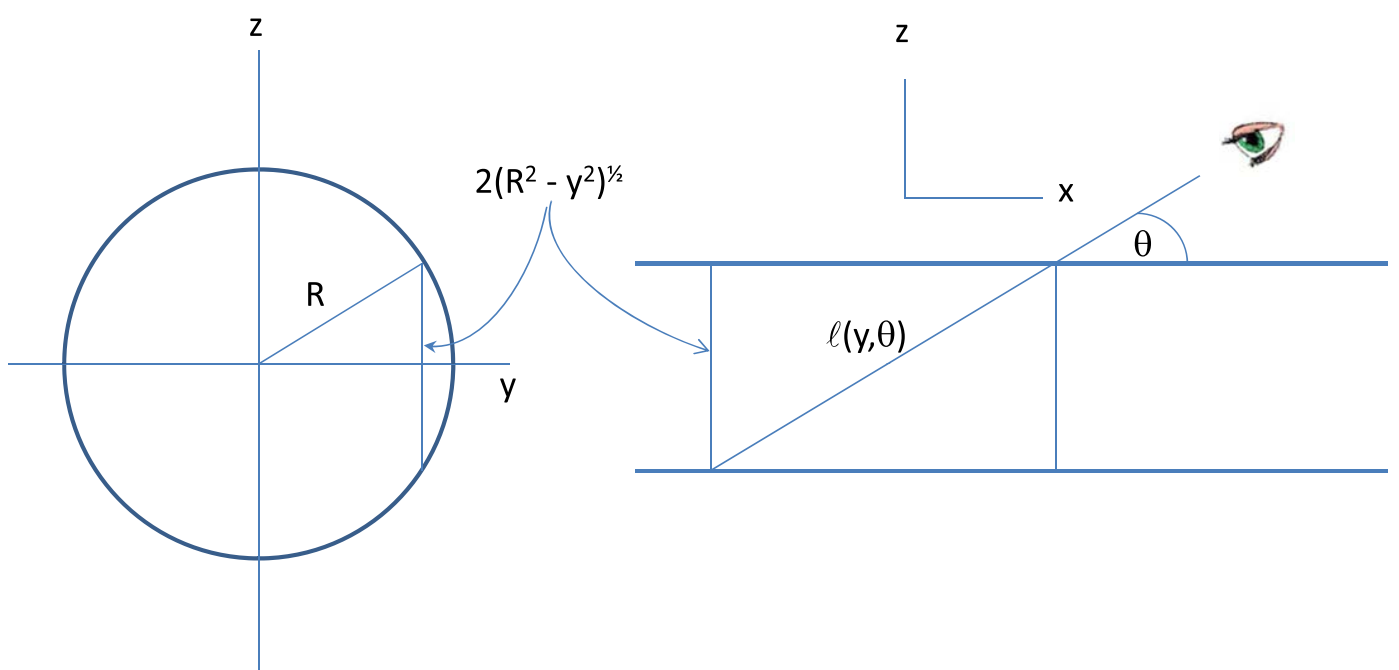

Figure 5. Filament viewed at angle $\theta$ along a path displaced by impact parameter $y$ from the axis. The cut on the left is perpendicular to the axis, and the one on the right is parallel to the axis and contains the viewing path.

the absorbing component, and denote by $S_{\nu}$ the intensity of emission that would have emerged in the absence of the absorbers. Assuming the emitters and absorbers to be well mixed together, the emission is everywhere proportional to the absorption so that the intensity generated between $\tau_{\nu}$ and $\tau_{\nu}+d \tau_{\nu}$ is $S_{\nu} d \tau_{\nu} / \tau_{\mathrm{T}_{\nu}}$. On its way out, this radiation is attenuated by $\exp \left(-\tau_{\nu}\right)$, emerging as $d I_{\nu}=S_{\nu} e^{-\tau_{\nu}} d \tau_{\nu} / \tau_{\mathrm{T}_{\nu}}$. Integrating over the path, the emerging intensity is now $I_{\nu}=S_{\nu} T_{\nu}$, instead of $S_{\nu}$, where

$$
T_{\nu}=\frac{1}{\tau_{\mathrm{T}_{\nu}}} \int e^{-\tau_{\nu}} d \tau_{\nu}=\frac{1-\exp \left(-\tau_{\mathrm{T}_{\nu}}\right)}{\tau_{\mathrm{T}_{\nu}}}
$$

is the transmission factor for the smooth-density absorbing component. This factor has the same functional form as the clumping factor $K_{\nu}$ (Equation (6)), approaching the limits $T_{\nu} \simeq 1$ when $\tau_{\mathrm{T}_{\nu}}<1$ and $T_{\nu} \simeq 1 / \tau_{\mathrm{T}_{\nu}}$ when $\tau_{\mathrm{T}_{\nu}}>1$. In the former case, the radiation emerges intact from the entire slab; in the latter, it emerges only from within $\sim 1$ optical depth from the surface, and the fractional thickness of this surface layer is $1 / \tau_{\mathrm{T}_{\nu}}$.

In the Condon et al. (1991) model, synchrotron emission $S_{\nu} \propto \nu^{-p}$, typically with $p \sim 0.7$, is attenuated by free-free absorbing gas well mixed together with the emitting gas. ${ }^{15}$ Emission from the free-free gas can be neglected at radio wavelengths so that the emergent radiation is just the absorbed synchrotron emission $I_{\nu}=S_{\nu} T_{\nu}$, where $T_{\nu}$ is the free-free transmission factor from Equation (41). Denote by $\nu_{0}$, the frequency where the free-free optical depth is unity, then $\tau_{\mathrm{T}_{\nu}}=\left(\nu_{0} / \nu\right)^{2.1}$ (see Equation (12)). At frequencies higher than $\nu_{0}, \tau_{\mathrm{T}_{\nu}}<1$ and the emergent intensity is $I_{\nu} \propto \nu^{-p}$, while at lower frequencies, $\tau_{\mathrm{T}_{\nu}}>1$ and $I_{\nu} \propto \nu^{2.1-p}$. Therefore, the spectrum peaks at $\nu_{0}$, falling away toward both lower and higher frequencies; this behavior is shown by the gray line in panel (a) of Figure 6.

We propose an extension of the Condon et al. (1991) model in which the synchrotron emission remains smoothly distributed but the free-free absorption is clumpy. This would be the

\footnotetext{
$\overline{15}$ A factor $1 / \tau_{f f}$ is missing from Equation (8) of Condon et al. (1991).
}

case, for instance, if the absorption came from individual $\mathrm{H}$ II regions within a star-forming region (Lacki 2013) while the synchrotron emission came from the interclump medium. As shown in Section 2, in this case, the attenuation is controlled by the effective optical depth $\tau_{\mathrm{E}}$ (Equation (1)). Assume that the density of the clumps along the LOS is always proportional to the synchrotron emission (because both are proportional to the star formation rate), and further assume that the clumping factor is constant along the LOS. Given these assumptions, exactly the same derivation as above for the smooth-absorption case is applicable, with $\tau_{\mathrm{E}}$ replacing $\tau_{\mathrm{T}}$ everywhere. The resulting emergent intensity again is $I_{\nu}=S_{\nu} T_{\nu}$, where now

$$
T_{\nu}=\frac{1-\exp \left(-\tau_{\mathrm{E}_{\nu}}\right)}{\tau_{\mathrm{E}_{\nu}}},
$$

with $\tau_{\mathrm{E}_{\nu}}$ the overall effective optical depth (Equation (1)). The smooth-absorption result in Equation (41) is recovered when $\tau_{\mathrm{E}_{\nu}}$ is replaced by the total optical depth $\tau_{\mathrm{T}_{\nu}}$; therefore, our extended model contains the Condon et al. (1991) model as a limiting case. Because $\tau_{\mathrm{E}_{\nu}}=K_{\nu} \tau_{\mathrm{T}_{\nu}}$ (Equation (5)), clumpiness affects the emergent radiation only at frequencies where the clumping factor $K_{\nu}$ deviates from unity, i.e., only at frequencies at which individual clumps are optically thick. In that case, $K_{\nu}<1$, and the mean effective optical depth is reduced because of the possibility that by chance, no optically thick clumps are encountered by radiation propagating out of the slab.

Now consider keeping the mean total LOS optical depth at the fiducial $\nu_{0}$ fixed at $\tau_{\mathrm{T}_{0}}=1$, with all this absorption concentrated into a varying number $\mathcal{N}$ of identical clouds. This corresponds to a situation in which the mean integrated freefree EM (in units $\mathrm{pc} \mathrm{cm}^{-6}$ ) per LOS is kept constant but the EM per clump varies. The emergent spectra are shown with solid colored lines in panel (a) of Figure 6, while panel (b) shows the spectral index of each model. The unique properties of clumpy absorption stand out immediately in the $\mathcal{N}=0.1$ plot-the input synchrotron radiation emerges unperturbed at all frequencies, even $\nu<\nu_{0}$, where $\tau_{\mathrm{T}_{\nu}}>1$. The reason is that in crossing the entire region, the radiation can avoid all clouds 


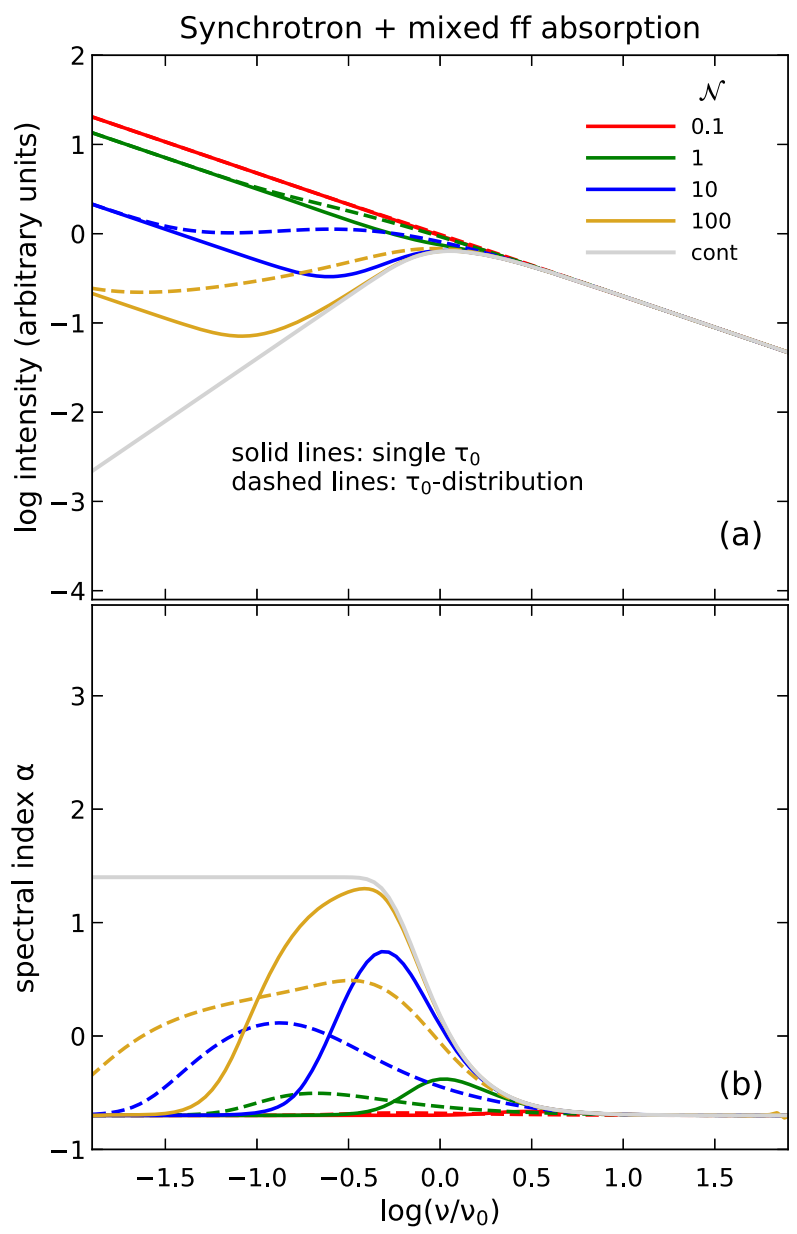

Synchrotron + foreground $\mathrm{ff}$ absorption

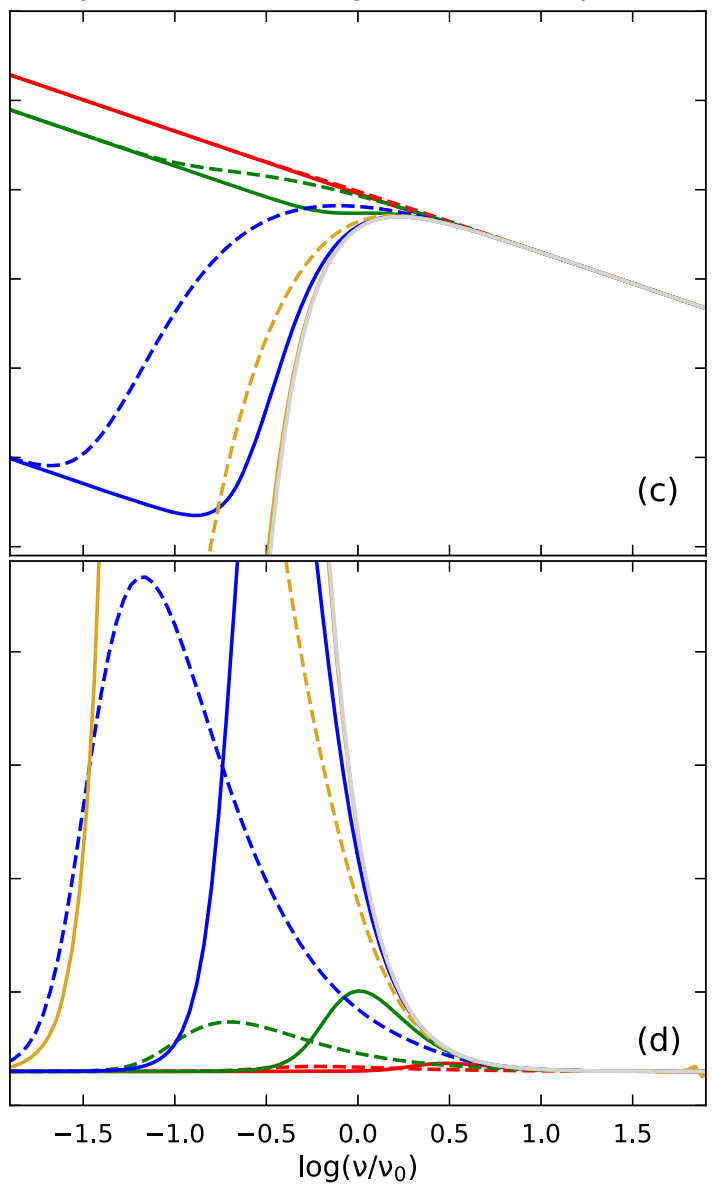

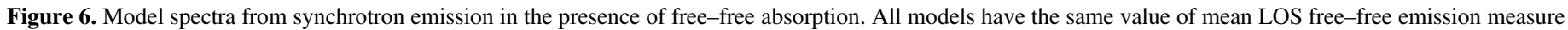

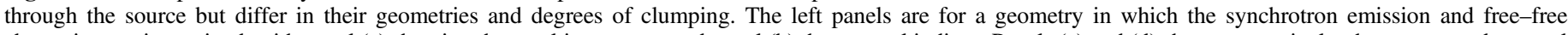

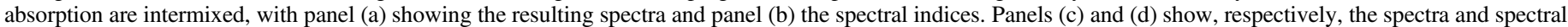

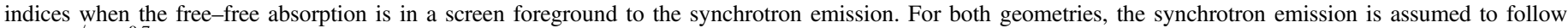

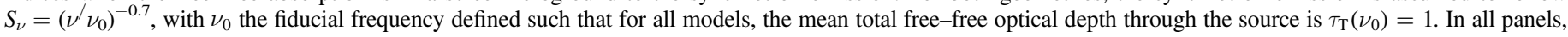

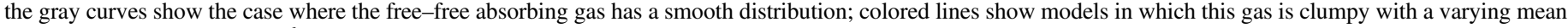

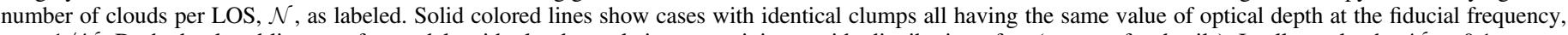

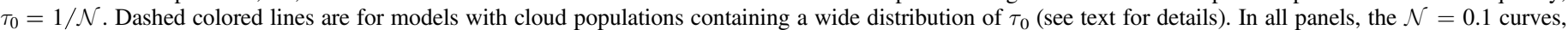

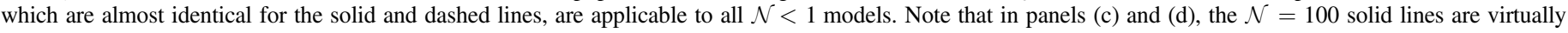
indistinguishable from the smooth-density absorption case.

with a probability $e^{-\mathcal{N}}$, which is $\simeq 1$ when $\mathcal{N}<1$. Thus, the $\mathcal{N}=0.1$ curve in Figure 6(a) is virtually identical to the input power-law spectrum and is representative of all $\mathcal{N}<1$ models. When $\mathcal{N}>1$, a fraction $1-e^{-\mathcal{N}}$ of the radiation will interact with the absorbing clouds, resulting in two spectral regimes depending on the optical depth $\tau_{\nu}$ of individual clouds. When $\tau_{\nu}<1$, clumping is irrelevant, and the emergent radiation is the same as for smooth-density absorption, while when $\tau_{\nu}>1$, $\tau_{\mathrm{E}_{\nu}}=\mathcal{N}$ (Equation (1)), the overall transmission factor is $T_{\nu} \simeq 1 / \mathcal{N}$ (Equation (42)), and the emergent intensity is $S_{\nu} / \mathcal{N}$. The observed radiation switches from the spectral shape of the smooth-absorption model to that of the input synchrotron, albeit at a reduced amplitude, at the frequency $\nu_{N}$ that gives $\tau\left(\nu_{N}\right)=1$ for each clump, i.e., a total optical depth $\tau_{\mathrm{T}_{\nu_{N}}}=\mathcal{N}$. Since $\tau_{\mathrm{T}_{\nu_{0}}}=1$, it follows that

$$
\nu_{N}=\frac{\nu_{0}}{\mathcal{N}^{0.48}}
$$

This transition is evident in the displayed cases of $\mathcal{N}=10$ and 100 , which have $\nu_{N} / \nu_{0}=0.33$ and 0.11 , respectively-at
$\nu>\nu_{N}$, the solutions overlap with the smooth-density result, while at $\nu<\nu_{N}$, the output spectrum equals the input synchrotron at reduced strength. As $\mathcal{N}$ is further increased, $\nu_{N}$ is decreasing, and the deviation from the smooth-density absorption is moving to the left. The smooth-absorption case is fully recovered in the formal limit $\mathcal{N} \rightarrow \infty$, where $\nu_{N} \rightarrow 0$ and all clouds are optically thin at all frequencies.

While the above discussion assumes that all the absorbing clumps are identical, the formalism can readily deal with the more realistic scenario of a wide distribution in clump properties. The dashed colored lines in Figure 6(a) show example spectra for such cases. As in the case of UCHIIs (Section 4.1), the distribution of clump optical depths at frequency $\nu_{0}$ follows Equation (13) with $\gamma=1.5$ and $\tau_{0, \max } / \tau_{0, \min }=10^{4}$. Different colored dashed lines again correspond to different choices for $\mathcal{N}$, the mean number of clumps per LOS. In each such case, the $\tau_{0 \text {,min }}$ of the clump opacity distribution is adjusted so that the mean total optical depth at $\nu_{0}$ averaged over all clumps is unity; i.e., for each $\mathcal{N}$, 
the value of $\tau_{0, \min }$ is adjusted so that, averaged over the $\tau_{0}$ distribution, the mean single-clump optical depth at the fiducial frequency $\nu_{0}$ is $\left\langle\tau_{0}\right\rangle=1 / \mathcal{N}$. Compared with the case of identical clumps (solid lines), in the clump distribution case, the frequency range over which the spectral index is flatter than the pure synchrotron spectrum is much wider, while the peak positive spectral index is reduced in value (see Figure 6(b)); these changes occur because the relatively sharp transition at $\nu=\nu_{N}$ (Equation (43)) for identical clumps is now spread over a range of frequencies. It is interesting to note that the flattened spectral shape of the $\mathcal{N}=10$ case in Figure 6(a) (blue dashed line) and the corresponding spectral index in Figure 6(b) are similar to some recent low-frequency observations of star-forming galaxies (Marvil et al. 2015; Calistro Rivera et al. 2017; Galvin et al. 2018). Clumpy free-free absorption with a wide range of clump opacities mixed with a synchrotron-emitting medium is thus a possible explanation. ${ }^{16}$

As with the original Condon et al. (1991) work, all model spectra presented here ignore contributions from free-free emission. For typical star formation-powered radio emission, where clump free-free absorption only becomes significant below $1 \mathrm{GHz}$, this is a good approximation up to $20-100 \mathrm{GHz}$. A more complete, integrated spectrum can be calculated by combining the model clump free-free emission spectrum from a population of clumps with the spectra described in Section 4.1 (and shown in Figure 3). We plan to present such overall spectra fitted to galaxy spectral energy distributions (SEDs) in a future publication.

\section{C.1. Absorption by Foreground Screen}

Observations of the evolving radio spectra of supernovae are broadly explained by synchrotron emission from the supernova shell that passes through a foreground free-free absorbing ionized wind from the progenitor star (Weiler et al. 1986, 2002). It is now thought that mass loss from such massive stellar progenitors is clumped (Smith 2014); indeed, observations of radio supernovae show evolving radio spectra that are not always compatible with a smooth progenitor wind (van Dyk et al. 1994; Weiler et al. 2002).

In calculating the intensity $I_{\nu}$ of background radiation that passes through foreground absorption, the only difference from the internal absorption discussed above is that the functional form of the transmission factor $T_{\nu}$ is replaced by an exponential with the same argument: $\tau_{\mathrm{T}_{\nu}}$ when the absorbing material is distributed smoothly (cf. Equation (41)), and $\tau_{\mathrm{E}_{\nu}}$ when it is clumpy (cf. Equation (42)). Therefore, in the case of smoothdensity absorption, the emerging spectra differ in the two scenarios only at $\nu<\nu_{0}$, where the foreground screen yields $I_{\nu}=S_{\nu} \exp \left(-\tau_{\mathrm{T}_{\nu}}\right)$ instead of $I_{\nu}=S_{\nu} / \tau_{\mathrm{T}_{\nu}}$ for internal obscuration and large $\tau_{\mathrm{T}_{\nu}}$. In the case of clumpy absorption with $\mathcal{N}<1$, the input radiation passes through almost unmodified irrespective of the geometry, thanks to the high probability to avoid all clouds. And when $\mathcal{N}>1$, clumpy absorption deviates from its smoothdensity counterpart only at $\nu<\nu_{N}$ (Equation (43)), where the foreground screen yields $I_{\nu}=S_{\nu} e^{-\mathcal{N}}$ instead of $S_{\nu} / \mathcal{N}$ for internal obscuration and large $\mathcal{N}$. Foreground screens can thus produce, for both smooth and clumpy absorption, much steeper spectral falloffs at low frequency than internal obscuration.

\footnotetext{
16 Alternative explanations include low-frequency modifications in the energy power-law index of lower-energy synchrotron-emitting electrons or spatially separated source components, which are free-free absorbed at different frequencies.
}

Panel (c) of Figure 6 shows synchrotron spectra attenuated by free-free absorbing foreground screens with both smooth and clumpy density distributions, repeating all the parameter combinations of the previous section; panel (d) shows the spectral index of each model. The above analysis is borne out by the numerical calculations. In particular, the synchrotron radiation emerges almost unmodified when $\mathcal{N}=0.1$; this happens in all $\mathcal{N} \ll 1$ models, whatever the geometrical configuration. And compared with the corresponding internalabsorption results, the spectral declines are much steeper for both smooth absorption at $\nu<\nu_{0}$ and $\mathcal{N}>1$ clumpy absorption at $\nu<\nu_{N}$. With the dynamic range covered in the figure, clumpy foreground screens with $\mathcal{N}>10$ (such as the displayed $\mathcal{N}=100$ ) are indistinguishable from smooth ones. As is evident from panels (b) and (d), a foreground screen can produce extreme cases of spectral index in comparison with internal obscuration.

The problem of synchrotron emission with free-free absorption contains two elements that combine into four possible configurations: the absorbing material can be internal or external to the emission region, and its distribution can be either smooth or clumpy. The literature on fitting the spectra and light curves of radio supernovae (e.g., Weiler et al. 2002) is not always clear on the distinctions between the different combinations and the origins of the factors accounting for the various types of absorption. As shown here, smooth-density absorption is described by the transmission factor $T\left(\tau_{\mathrm{T}_{\nu}}\right)$ (Equation (41)) when it is internal and $\exp \left(-\tau_{\mathrm{T}_{\nu}}\right)$ when it is external. When the absorbing material is clumped, the overall optical depth $\tau_{\mathrm{T}_{\nu}}$ is simply replaced in either case by the effective optical depth $\tau_{\mathrm{E}_{\nu}}$ (Equation (1)). And since $\tau_{\mathrm{E}_{\nu}}=$ $K_{\nu} \tau_{\mathrm{T}_{\nu}}$ (Equation (5)), the only effect of clumpiness is to modify the optical depth by a clumping correction factor $K_{\nu}$ (Equation (6)), whether or not the extinction is internal or external.

\section{ORCID iDs}

Moshe Elitzur (iD https://orcid.org/0000-0001-8143-3550

\section{References}

Calistro Rivera, G., Williams, W. L., Hardcastle, M. J., et al. 2017, MNRAS, 469, 3468

Condon, J. J., Huang, Z.-P., Yin, Q. F., \& Thuan, T. X. 1991, ApJ, 378, 65

Elitzur, M. 1992, Astronomical Masers (Dordrecht: Kluwer)

Elmegreen, B. G., \& Scalo, J. 2004, ARA\&A, 42, 211

Falgarone, E., Phillips, T. G., \& Walker, C. K. 1991, ApJ, 378, 186

Galvin, T. J., Seymour, N., Marvil, J., et al. 2018, MNRAS, 474, 779

Ignace, R., \& Churchwell, E. 2004, ApJ, 610, 351

Lacki, B. C. 2013, MNRAS, 431, 3003

Laor, A., Barth, A. J., Ho, L. C., \& Filippenko, A. V. 2006, ApJ, 636, 83

Martin, H. M., Hills, R. E., \& Sanders, D. B. 1984, MNRAS, 208, 35

Marvil, J., Owen, F., \& Eilek, J. 2015, AJ, 149, 32

Natta, A., \& Panagia, N. 1984, ApJ, 287, 228

Nenkova, M., Ivezić, Z., \& Elitzur, M. 2002, ApJL, 570, L9

Nenkova, M., Sirocky, M. M., Ivezić, Z., \& Elitzur, M. 2008, ApJ, 685, 147

Pirogov, L. E., Zinchenko, I. I., Johansson, L. E. B., \& Yang, J. 2012, A\&AT, 27,475

Smith, N. 2014, ARA\&A, 52, 487

Taine, J., Iacona, E., \& Bellet, F. 2008, in 5th European Thermal-Sciences Conf., EUROTHERM 2008, ed. G. G. M. Stoffels, T. H. van der Meer, \& A. A. van Steenhoven (Eindhoven: EUROTHERM), hal-00340096 Tauber, J. A. 1996, A\&A, 315, 591

Tauber, J. A., Goldsmith, P. F., \& Dickman, R. L. 1991, ApJ, 375, 635 
Thronson, H. A., Jr., Majewski, S., Descartes, L., \& Hereld, M. 1990, ApJ, 364,456

van Dyk, S. D., Weiler, K. W., Sramek, R. A., Rupen, M. P., \& Panagia, N. 1994, ApJL, 432, L115

Wall, W. F. 2006, RMxAA, 42, 117

Wall, W. F. 2007, MNRAS, 379, 674
Weiler, K. W., Panagia, N., Montes, M. J., \& Sramek, R. A. 2002, ARA\&A, 40, 387

Weiler, K. W., Sramek, R. A., Panagia, N., van der Hulst, J. M., \& Salvati, M. 1986, ApJ, 301, 790

Weiler, K. W., van Dyk, S. D., Sramek, R. A., \& Panagia, N. 2004, NewAR, 48, 1377 